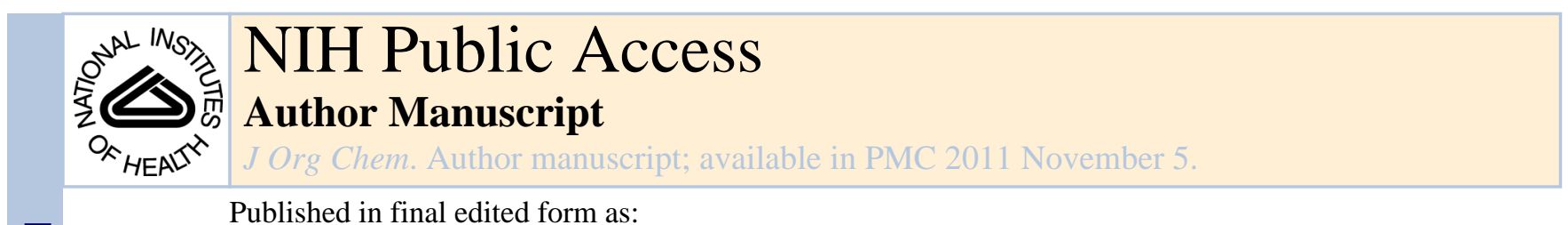

Published in final edited form as:

J Org Chem. 2010 November 5; 75(21): 7052-7060. doi:10.1021/jo101598y.

\title{
A Total Synthesis of (-)-Callipeltoside A
}

\author{
Thomas R. Hoye ${ }^{*}$, Michael E. Danielson, Aaron E. May, and Hongyu Zhao \\ Department of Chemistry, University of Minnesota, Minneapolis, Minnesota 55455
}

\begin{abstract}

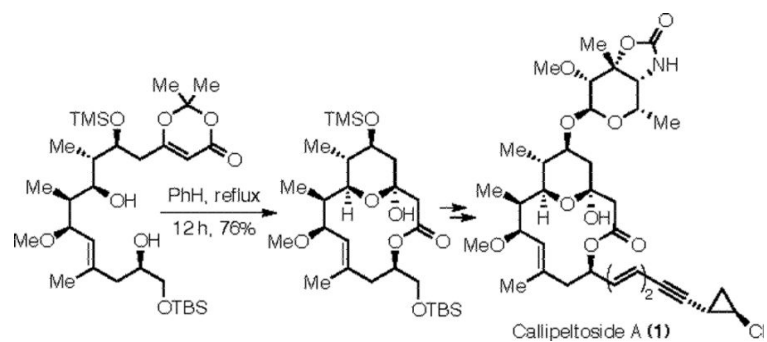

A total synthesis of (-)-callipeltoside A (1) has been achieved. The core macrocycle was made via a dual macrolactonization/pyran hemiketal formation reaction, which was developed to circumvent issues related to the reversible nature of acylketene formation from $\beta$-keto lactone substrates. Initial approaches to the core of the natural product that revolved around ring-closing metathesis $(\mathrm{RCM})$ and relay ring-closing metathesis (RRCM) reactions are also described.
\end{abstract}

\section{Introduction}

Callipeltoside A (1) is a cytotoxic macrolide isolated in 1996 from the marine lithistida sponge (Callipelta sp.). ${ }^{1}$ The stereochemical features of callipeltoside A were deduced on the basis of extensive NMR studies. When we initiated the research described here, issues still remained with respect to i) the absolute configuration and ii) the relative configuration of $C(13)$ and $C(20) / C(21)$ vis-à-vis those in the remainder of the molecule. The relative configuration of $\mathrm{C}(13)$ was established by Paterson and coworkers. ${ }^{2}$ The first total synthesis, which established the remaining relative configurational issues of $\mathrm{C}(20) / \mathrm{C}(21)$ as well as the overall absolute configuration of (-)-callipeltoside A (1) was reported by the Trost labs in 2002. ${ }^{3 \mathrm{a}}$ Additional total syntheses have been reported, ${ }^{3 \mathrm{~b}-\mathrm{g}}$ and numerous related studies have appeared. ${ }^{4}$

\section{Results/Discussion}

Our approach to $\mathbf{1}$ involved a convergent union of the three key intermediates: macrolactone $\mathbf{2}$, carbohydrate $\mathbf{3}$, and phosphonate $\mathbf{4}$ (Scheme 1). Our initial approach to the synthesis of $\mathbf{2}$ aimed to utilize a RCM reaction from a suitable precursor, such as $\mathbf{5} .{ }^{5}$ Unfortunately, neither $\mathbf{5}$ or its $\mathrm{C}(13)$ epimer (epi-5) could be ring-closed to give a macrocyclic product (Scheme 2). Using Grubbs' first generation metathesis initiator (7), ${ }^{6}$ no initiation was observed at room temperature, as evidenced by lack of styrene formation by analysis of the NMR spectrum and recovery of starting material. Attempts to alter the reactivity of the substrate by using a more constrained, and perhaps geometrically favorable, conformer (cf. 6) also failed. ${ }^{7}$ At

\footnotetext{
*hoye@umn.edu .

Supporting Information Available: Experimental procedures, characterization data, and copies of ${ }^{1} \mathrm{H}$ and ${ }^{13} \mathrm{C}$ NMR spectra for new compounds (182 pages). This material is available free of charge via the Internet at http://pubs.acs.org.
} 
elevated temperatures $\left(50-70{ }^{\circ} \mathrm{C}\right.$, benzene solvent) the catalyst [either 7 or the second generation initiator $(\mathbf{8})]^{8}$ decomposed and starting material (5-6) could be recovered.

Believing that the main problem with the lack of reactivity of substrates 5 and $\mathbf{6}$ was due to slow initiation, we hypothesized that the use of a relay strategy would be advantageous. ${ }^{5 b, 9}$ The concept, outlined in Scheme 3, was to use allyloxymethyl relays on substrates 9 and $\mathbf{1 0}$. Ruthenium loading onto the terminal alkene of the relay was envisioned to give intermediates 11 and 12. Intramolecular cyclization of each would give 13 or 14, respectively, with concomitant formation of 3,4-dihydrofuran. Finally, cyclization of $\mathbf{1 3}$ and $\mathbf{1 4}$ would give the desired $\beta$-keto lactone $\mathbf{1 5}$ and regenerate a ruthenium methylidene as the active catalyst. Since we had no way of knowing whether intermediate $\mathbf{1 3}$ or $\mathbf{1 4}$ would be more likely to macrocyclize, we chose to undertake the synthesis of both substrates $\mathbf{9}$ and 10.

The synthesis of relay substrate $\mathbf{9}$ is outlined in Scheme 4. Known Weinreb amide $\mathbf{1 6}$ was protected as a PMB ether using PMB-trichloroacetimidate and catalytic CSA. ${ }^{10}$ Hydroboration/oxidation (9-BBN), followed by TBS protection and DIBAL-H reduction of the Weinreb amide gave aldehyde 17. Lithium-halogen exchange of alkenyl iodide $\mathbf{1 8}$ followed by addition to aldehyde $\mathbf{1 7}$ gave a 1:1.5 ratio of newly formed secondary alcohols. Subsequent methylation with methyl iodide gave the relay-armed substrate 19. Deprotection of the primary TBS ether using TBAF, oxidation with Dess-Martin periodinane, vinylogous Mukaiyama aldol reaction with diene 20, 11 and TMS protection gave dioxinone substrate 21. Refluxing this substrate in benzene with secondary alcohol $\mathbf{2 2}, 12$ followed by TMS deprotection with PPTS in acetonitrile gave $\mathbf{9}$.

The synthesis of the alternative relay substrate $\mathbf{1 0}$ is outlined in Scheme 5. The allyl ether of known bromo alkene $\mathbf{2 3}^{13}$ was used, after lithium-halogen exchange (cat. CuI), to ring-open the TBS protected epoxide $\mathbf{2 4}^{14}$ to give relay-armed alcohol $\mathbf{2 5}$. Aldehyde $\mathbf{1 7}$ was elaborated by addition of vinyl magnesium bromide, giving a separable mixture of diastereomeric ( $\mathrm{dr}$ 1:1) alcohols. Methylation with methyl iodide, TBAF deprotection, and oxidation with Dess-Martin periodinane gave aldehyde 26. The vinylogous Mukaiyama aldol reaction of $\mathbf{2 6}$ with diene $\mathbf{2 0}$ and TMS protection of the C(5)-hydroxyl gave dioxinone 27. Refluxing a mixture of alcohol 25 and dioxinone $\mathbf{2 7}$ in benzene, followed by TMS deprotection, gave relay substrate $\mathbf{1 0 .}$

Unfortunately for our cause, subjection of either $\mathbf{9}$ or $\mathbf{1 0}$ to the metathesis initiator $\mathbf{8}$ did not lead to the formation of desired product $\mathbf{1 5}$, but instead gave $\mathbf{5}$, the substrate for the initial RCM study. The process leading to $\mathbf{5}$ is outlined in Scheme 6; an event we refer to as truncation. When metathesis initiator $\mathbf{8}$ reacts with 10, the relay to produce $\mathbf{2 8}$ presumably proceeds as planned, as evidenced by formation of 3,4-dihydrofuran ( ${ }^{1} \mathrm{H}$ NMR). In order for macrocyclic ring closure to occur within $\mathbf{2 8}$, the rate of its intramolecular cyclization $\left(k_{\text {intra }}\right)$ must be faster than the rate of bimolecular attack on the more reactive alkene in another molecule of $\mathbf{1 0}\left(k_{\text {inter }}\right)$. Since truncation is bimolecular, we recognized that running the reaction at higher dilution would result in a more favorable $k_{\text {intra }} / k_{\text {inter }}$ ratio, but even at $10^{-5} \mathrm{M}$, only the truncation product 5 was observed. ${ }^{7}$

We examined other ways to favorably bias the ratio of $k_{\text {intra }}$ to $k_{\text {inter }}$ during this RRCM reaction. Since disubstituted alkenes are slower to engage in cross-metathesis (CM), it was hoped that using a 1,1-disubstituted alkene within the relay arm would slow the bimolecular truncation rate sufficiently so that macrocyclization could prevail. To this end, substrates $\mathbf{2 9}$ and 30 (Scheme 7) were synthesized in an analogous manner. While our reasoning was apparently sound, as evidenced by a significantly slower overall rate of reaction, no desired product was formed using these substrates having the modified relay subunit. Instead the 
truncation products $\mathbf{5}$ and $\mathbf{6}$ were observed, respectively. The clear implication is that the initial relay event occurred to deliver ruthenium to the more hindered carbon atom of the otherwise inactive alkene. As these results illustrate, the use of a RRCM strategy by no means guarantees a successful outcome. If the inherent rate of the crucial metathesis cyclization event is slower than competing events (e.g, $\mathrm{CM}$ or $[\mathrm{Ru}]$ decomposition), as is the case here, then the cyclization will not be efficient regardless of the process by which the key ruthenium alkylidene intermediate is formed. ${ }^{9 c}$

We then turned to a strategy in which the Boeckman lactonization ${ }^{15}$ of an acylketene intermediate would be used to close the core macrocycle. ${ }^{5 \mathrm{~b}}$ This strategy formed the bases of both the Trost synthesis of $\mathbf{1}^{3 \mathrm{a}, \mathrm{c}}$ and the Marshall synthesis ${ }^{16}$ of the aglycone. Reaction of the TBS protected epoxide $\mathbf{3 1}$ with lithium TMS-acetylide, followed by a two-step deprotection gave diol 32. ${ }^{17,18}$ Negishi carboalumination/iodination ${ }^{19}$ and sequential protection of the 1,2-diol as TBS and PMB ethers gave 33. Lithium-halogen exchange in $\mathbf{3 3}$ and addition to aldehyde $\mathbf{3 4}$ gave a 1:1 mixture of separable secondary alcohols. ${ }^{20}$ Recycling of the undesired epimer by oxidation with Dess-Martin periodinane, followed by reduction with lithium triethylhydridoborate gave the desired epimer in a 10:1 dr. Subsequent methylation gave alkene 35, which was subjected to hydroboration/oxidation (9-BBN). The resulting primary alcohol was subjected to Dess-Martin periodinane oxidation and vinylogous Mukaiyama aldol reaction with diene 20. Each of the two new stereocenters at $\mathrm{C}(5)$ and $\mathrm{C}(6)$ were formed with essentially full diastereocontrol. Protection of the $\mathrm{C}(5)-\mathrm{OH}$ with $\mathrm{MOMCl}$ and $\mathrm{PMB}$ removal at $\mathrm{C}(13)$ with DDQ gave the lactonization precursor 36.

The dioxinone-containing substrate $\mathbf{3 6}$ was heated in refluxing toluene to effect thermal extrusion of acetone and concomitant generation of the acylketene 37. Macrolactonization involves cylization of the $\mathrm{C}(13)$ hydroxyl group to give enol 38. Two products were isolated: the keto lactone 39 and the methyl ketone $\mathbf{4 0}$ (64\% and 15\%, respectively) (Scheme 9). The latter arises from competitive addition of water to $\mathbf{3 7}$ and subsequent decarboxylation of the resulting $\beta$-ketoacid, a phenomenon that has been observed by others. ${ }^{21}$ The relative amount of $\mathbf{4 0}$ increased over time. This suggests that the unimolecular lactonization of the acylketene $\mathbf{3 7}$ outcompetes its trapping by water and that the ketolactone $\mathbf{3 9}$ is able to revert via $\mathbf{3 8}$ to acylketene $37 .{ }^{22}$ While concentration effects are undoubtedly also important, it is relevant that i) addition of an $\mathrm{O}-\mathrm{H}$ bond to an acylketene is a concerted event (cf., 37 to 38 ${ }^{23}$ and ii) the $\mathrm{O}-\mathrm{H}$ bond dissociation energy of water is substantially higher than those of alcohols (119 vs. 104-107 $\left.\mathrm{kcal} \mathrm{mol}^{-1}\right){ }^{24}$ We hypothesized that the detrimental outcome of competitive water addition could be mitigated if the $\beta$-ketone in a macrolactone like 39 could be sequestered. An attractive possibility was that it be trapped as its corresponding pyran-hemiketal by a free hydroxyl group at $\mathrm{C}(7){ }^{22}$

To test this idea, we elected to prepare diol $\mathbf{4 3}$ (Scheme 10). ${ }^{25}$ Thus, the vinyllithium species derived from ent-33 was added to aldehyde $\mathbf{4 1}$ to give a 1:1 ratio of diols. Separation and methylation with methyl iodide gave alkene 42. Hydroboration/oxidation, Dess-Martin oxidation, and vinylogous Mukaiyama aldol reaction with diene $\mathbf{2 0}$ was performed. Protection of the resulting secondary alcohol, followed by DDQ removal of both PMB ethers afforded the dioxinone diol $\mathbf{4 3}$.

To our delight, when refluxed in benzene for 12 hours, dioxinone $\mathbf{4 3}$ produced the desired dual macrocyclic pyran-hemiketal $\mathbf{4 4}$ in $76 \%$ yield (Scheme 11). We suspect that pyranhemiketal formation within either enol $\mathbf{4 6}$ or ketone $\mathbf{4 7}$ follows macrolactonization (45 to 46). It is noteworthy that no products indicative of competitive trapping of the acylketene 45 by water could be detected (and no 8-membered lactone from reaction of the $\mathrm{C}(7)-\mathrm{OH}$ with the ketene carbon was observed). It is clear that in situ protection of the $\mathrm{C}(3)$-ketone as its hemiketal is advantageous. ${ }^{22}$ 
The completion of the synthesis, following established lines, is outlined in Scheme 12. A three-step protecting group manipulation was used to convert 44 into 48. Parikh-Doerin'g oxidation afforded the corresponding aldehyde, from which aglycone $\mathbf{4 9}$ was obtained by Horner-Emmons olefination with the known phosphonate $4,{ }^{26}$ followed by deprotection of the TMS ether at $\mathrm{C}(5)$. Glycosidation ${ }^{3 \mathrm{a}}$ with known acetimidate $\mathbf{3}^{27}$ provided (-)callipeltoside A (1).

In conclusion, we have completed a total synthesis of (-)-callipeltoside A (1). Initial attempts using an RCM reaction on substrates $\mathbf{5}$ and $\mathbf{6}$ were not successful; presumably these compounds are simply too hindered to load ruthenium to the requisite (and more hindered) alkene carbon atom. This led to the evolution of the RRCM strategy, which was explored via substrates $9,10,29$, and 30 . In each case, relay activation allowed ruthenium to be transiently installed to the necessary site (cf. 13, 14, and 28), but bimolecular truncation of these species thwarted the (inherently slow) final ring-closing event. Ultimately, the synthesis of 1 (20 linear steps from TBS-glycidol 24, 0.7\% yield) was enabled by a highly efficient acylketene mediated dual macrolactonization/pyran-hemiketal formation reaction, converting the linear precursor $\mathbf{4 3}$ directly to the bridged bicyclic macrolactone $\mathbf{4 4}$.

\section{Experimental Section}

General. ${ }^{1} \mathrm{H}$ NMR chemical shifts in $\mathrm{CDCl}_{3}$ are referenced to TMS $(0.00 \mathrm{ppm})$ and in $\mathrm{PhH}-$ $d_{6}$ to 7.16. ${ }^{13} \mathrm{C}$ NMR chemical shifts in $\mathrm{CDCl}_{3}$ are referenced to chloroform (77.2 ppm). The following format has been used to report peaks: chemical shift in ppm [multiplicity, coupling constant(s) in $\mathrm{Hz}$, integral]. MPLC refers to medium pressure liquid chromatography (25-60 psi) using hand-packed columns of E. Merck silica gel (230-400 mesh), a pump, and a differential refractive index detector. Reactions requiring anhydrous conditions were performed under an atmosphere of nitrogen or argon in oven-dried glassware. Dry diethyl ether and benzene were distilled from sodium benzophenone ketyl. Toluene was distilled from Na/K. Anhydrous THF and methylene chloride were passed through a column of activated alumina. Triethylamine and pyridine were distilled from $\mathrm{KOH}$ just prior to use. DMF was stored over 4 Angstrom molecular sieves. The structure of each intermediate numbered here as $\mathbf{S} \#$ can be found in the Supporting Information.

\section{(+)-(2S)-4-Pentyne-1,2-diol (32) $)^{28}$}

To epoxide 31 (13.3 g, $71.0 \mathrm{mmol})$, TMS-acetylene ( $15 \mathrm{~mL}, 106.0 \mathrm{mmol})$, and THF (141 $\mathrm{mL}$ ) was added $n$-BuLi $(3.2 \mathrm{M}$ in hexanes, $40.0 \mathrm{~mL}, 92.0 \mathrm{mmol})$ dropwise at $-78{ }^{\circ} \mathrm{C}$, followed by $\mathrm{BF}_{3} \cdot \mathrm{OEt}_{2}(13.4 \mathrm{~mL}, 106.0 \mathrm{mmol})$ dropwise at the same temperature. The solution was stirred for $45 \mathrm{~min}$ at $-78{ }^{\circ} \mathrm{C}$ before being quenched with aqueous $\mathrm{NH}_{4} \mathrm{Cl}(20$ $\mathrm{mL})$. The mixture was allowed to warm to rt and diluted with $\mathrm{H}_{2} \mathrm{O}(150 \mathrm{~mL})$. The aqueous layer was extracted with DCM $(3 \times 100 \mathrm{~mL})$ and the combined organic portions were dried over $\mathrm{Na}_{2} \mathrm{SO}_{4}$ and concentrated to give crude TMS alkyne as a colorless oil. This alkyne was immediately subjected to desilylation. To the crude oil was added $\mathrm{K}_{2} \mathrm{CO}_{3}(29.3 \mathrm{~g}, 212 \mathrm{~mol})$ and $\mathrm{MeOH}(100 \mathrm{~mL})$, and this mixture was stirred for $24 \mathrm{~h}$ at $\mathrm{rt}$. The suspended $\mathrm{K}_{2} \mathrm{CO}_{3}$ was filtered through celite on a sintered glass funnel with $\mathrm{MeOH}$. The filtrate was concentrated to afford the crude alkyne as a mixture of primary and secondary TBS ethers. The crude products were carried into the next step without purification. To the mixture of alkynes was added $\mathrm{MeOH}(40 \mathrm{~mL})$, THF $(40 \mathrm{~mL})$, and $\mathrm{KF}(24 \mathrm{~g}, 420 \mathrm{mmol})$; and the resulting slurry was refluxed at $65{ }^{\circ} \mathrm{C}$ for $48 \mathrm{~h}$. The mixture was cooled to rt, concentrated, placed on a flash column, and purified via flash chromatography (hexanes:EtOAc $=1: 1$ followed by $100 \%$ EtOAc) to provide a pale yellow oil that crystallized in a freezer overnight to afford diol $\mathbf{3 2}$ as white needles $(3.2 \mathrm{~g}, 77 \%) .{ }^{1} \mathrm{H}$ NMR $\left(300 \mathrm{MHz}, \mathrm{CDCl}_{3}\right) \delta 3.91$ (dddd, $J=3.7,6.2,6.2$, and $6.2 \mathrm{~Hz}, 1 \mathrm{H}), 3.77(\mathrm{dd}, J=3.7$ and $11.1 \mathrm{~Hz}, 1 \mathrm{H}), 3.62(\mathrm{dd}, J=6.2$ and $10.0 \mathrm{~Hz}, 1 \mathrm{H}), 2.45$ (dd, $J=2.7$ and $6.2 \mathrm{~Hz}, 2 \mathrm{H}), 2.35(\mathrm{bs}, 1 \mathrm{H}), 1.90(\mathrm{bs}, 1 \mathrm{H})$, and $2.08(\mathrm{t}, J=2.7 \mathrm{~Hz}, 1 \mathrm{H}) .{ }^{13} \mathrm{C}$ 
NMR $\left(125 \mathrm{MHz}, \mathrm{CDCl}_{3}\right) \delta 80.3,70.7,70.2,65.3$, and 23.2. GC-MS $\mathrm{T}_{\mathrm{R}}=2.66 \mathrm{~min}$.; $\mathrm{m} / \mathrm{z}$ : 100,69 , and 61. IR (neat) 3300, 3292, 2113, 1719, 1416, 1085, and $1044 \mathrm{~cm}^{-1}$. mp 44-51 ${ }^{\circ} \mathrm{C}$. TLC $\mathrm{R}_{\mathrm{f}}=0.35,100 \%$ EtOAc. $[\alpha]^{\mathrm{RT}}+8.22^{\circ}\left(\mathrm{c}=1.1, \mathrm{CHCl}_{3}\right)$.

\section{(-)-(2S,4E)-1-[[(1,1-Dimethylethyl)dimethylsilyl]oxy]-5-iodo-2-[(4- methoxyphenyl)methoxy]-4-methyl-4-pentene (33)}

To zirconocene dichloride $(2.5 \mathrm{~g}, 9.6 \mathrm{mmol})$ and 1,2-dichloroethane $(90 \mathrm{~mL})$ was added $\mathrm{AlMe}_{3}(2.0 \mathrm{M}$ in toluene, $36 \mathrm{~mL}, 72 \mathrm{mmol})$ dropwise at rt followed by diol $32(2.4 \mathrm{~g}, 24$ mmol) in 1,2-dichloroethane $(20 \mathrm{~mL})$. The solution was stirred at $50{ }^{\circ} \mathrm{C}$ for $48 \mathrm{~h}$ and then cooled to $-40^{\circ} \mathrm{C}$. Iodine $(9.1 \mathrm{~g}, 36 \mathrm{mmol})$ in THF $(26 \mathrm{~mL})$ was added dropwise by cannula. The resulting dark brown solution was allowed to warm and was stirred for $30 \mathrm{~min}$ at $\mathrm{rt}$ before being poured into a mixture of hexanes and sodium potassium tartrate. The aqueous layer was extracted with EtOAc $(1 \times 50 \mathrm{~mL})$, saturated with $\mathrm{NaCl}$, and extracted again with EtOAc $(3 \times 50 \mathrm{~mL})$. The combined organic layers were dried over $\mathrm{Na}_{2} \mathrm{SO}_{4}$, concentrated, and chromatographed $(100 \%$ EtOAc) to provide iodo diol $(\mathrm{Rf}=0.13$ in $1: 1$ hexanes:EtOAc) as a pale yellow oil $(3.4 \mathrm{~g}, 58 \%)$. The diol $(3.0 \mathrm{~g}, 12.4 \mathrm{mmol})$ was dissolved in DCM (120 $\mathrm{mL})$ and treated with TEA $(7.0 \mathrm{~mL}, 50.0 \mathrm{mmol})$, DMAP $(230 \mathrm{mg} 1.86 \mathrm{mmol})$, and TBSCl ( $3.0 \mathrm{~g} 20.0 \mathrm{mmol})$. The resulting solution was stirred $24 \mathrm{~h}$ at $\mathrm{rt}$ before being quenched with aqueous $\mathrm{NH}_{4} \mathrm{Cl}(50 \mathrm{~mL})$, diluted with $\mathrm{H}_{2} \mathrm{O}(100 \mathrm{~mL})$ and extracted with DCM. The combined organic layers were dried over $\mathrm{Na}_{2} \mathrm{SO}_{4}$, concentrated, and chromatographed (hexanes:EtOAc $=19: 1)$ to provide a primary TBS ether $(\mathrm{Rf}=0.25$ in 9:1 hexanes:EtOAc) as a colorless oil ( $3.2 \mathrm{~g}, 72 \%)$. To this ether $(310 \mathrm{mg}, 0.87 \mathrm{mmol})$ in DCM $(5 \mathrm{~mL})$ was added para-methoxybenzyl trichloroacetimidate $(762 \mathrm{mg}, 2.7 \mathrm{mmol}$ ) and CSA (46 mg, 0.2 $\mathrm{mmol})$. The reaction mixture was stirred for $48 \mathrm{~h}$ before being quenched with aqueous $\mathrm{NaOH}(10 \%, 20 \mathrm{~mL})$. The mixture was extracted with DCM, washed with aqueous $\mathrm{NaOH}$ $\left(10 \%, 20 \mathrm{~mL}\right.$ ), dried over $\mathrm{MgSO}_{4}$, and concentrated. Purification by MPLC (hexanes:EtOAc $=12: 1)$ gave $33(330 \mathrm{mg}, 82 \%)$ as a colorless oil. ${ }^{1} \mathrm{H}$ NMR $\left(500 \mathrm{MHz}, \mathrm{CDCl}_{3}\right) \delta 7.23(\mathrm{~d}, J=$ $8.5 \mathrm{~Hz}, 2 \mathrm{H}), 6.89(\mathrm{~d}, J=8.5 \mathrm{~Hz}, 2 \mathrm{H}), 5.97(\operatorname{broad~s}, 1 \mathrm{H}), 4.60(\mathrm{~d}, J=11.5 \mathrm{~Hz}, 1 \mathrm{H}) ; 4.46(\mathrm{~d}$, $J=11.0 \mathrm{~Hz}, 1 \mathrm{H}), 3.81(\mathrm{~s}, 3 \mathrm{H}), 3.67(\mathrm{~m}, 1 \mathrm{H}), 3.55(\mathrm{dd}, J=5.0$ and $8.5 \mathrm{~Hz}, 1 \mathrm{H}), 3.52(\mathrm{dd}, J=$ 5.5 and $8.5 \mathrm{~Hz}, 1 \mathrm{H}), 2.44(\mathrm{dd}, J=3.5$ and $14.0 \mathrm{~Hz}, 1 \mathrm{H}), 2.36(\mathrm{dd}, J=8.0$ and $13.0 \mathrm{~Hz}, 1 \mathrm{H})$, 1.78 (broad s, 3H), 0.90 (s, 9H), and 0.06 (s, 6H). $\left.{ }^{13} \mathrm{C} \mathrm{NMR} \mathrm{(125} \mathrm{MHz,} \mathrm{CDCl}_{3}\right) \delta 159.2$, $144.9,130.6,129.5,113.8,77.2,77.1,72.0,65.0,55.3,41.8,25.9,24.3,18.3,-5.32$, and -5.37 . IR (neat) $3063,3032,2996,2958,2931,2857,1612,1512$, and $1250 \mathrm{~cm}^{-1}$. GC-MS $\mathrm{TR}=12.6 \mathrm{~min}$; $\mathrm{m} / \mathrm{z}: 419,349$, and 121. HRMS (CI, $4 \% \mathrm{NH}_{3}$ in $\left.\mathrm{CH}_{4}\right)$ Calcd for $\left(\mathrm{C}_{20} \mathrm{H}_{33} \mathrm{IO}_{3} \mathrm{Si}+\mathrm{NH}_{4}\right)^{+}:$494.1582. Found: 494.1579. TLC R $\mathrm{f}=0.3$, hexanes:EtOAc $=15: 1$. $[\alpha]^{\mathrm{RT}}-15.3^{\circ}\left(\mathrm{c}=1.0, \mathrm{CHCl}_{3}\right)$.

\section{(-)-[3S-(3 $\left.\left.R^{\star}, 4 S^{\star}, 5 S^{\star}, 9 S^{\star}\right), 6 E\right]-5-M e t h o x y-3,9-B i s-[(4-m e t h o x y p h e n y l) m e t h o x y]-2,4,7-$ trimethyl-10-[[(1,1-dimethylethyl)dimethylsilyl]oxy]-1,6-decadiene (42)}

To vinyl iodide ent-33 $(600 \mathrm{mg}, 1.26 \mathrm{mmol}$, prepared by the same sequence described above) and diethyl ether $\left(10 \mathrm{~mL}\right.$, stirred over $\mathrm{CaH}_{2}$ for $\left.1 \mathrm{~h}\right)$ in a round bottom flask was added solid $\mathrm{CaH}_{2}(5 \mathrm{mg})$, and the mixture was stirred for $10 \mathrm{~min}$ at $\mathrm{rt}$. The cloudy solution was cooled to $-78{ }^{\circ} \mathrm{C}$ and $n$-BuLi $(2.1 \mathrm{M}$ in hexane, $0.66 \mathrm{~mL}, 1.38 \mathrm{mmol})$ was added dropwise and stirred for $10 \mathrm{~min}$. At the same temperature aldehyde $\mathbf{4 1}^{29}$ (342 $\mathrm{mg}, 1.38$ $\mathrm{mmol})$ in diethyl ether $(2 \mathrm{~mL})$ was added to the flask by cannula, and the solution was stirred for $15 \mathrm{~min}$ before warming to $0{ }^{\circ} \mathrm{C}$. The reaction mixture was poured into a separatory funnel containing aqueous $\mathrm{NH}_{4} \mathrm{Cl}$, diluted with water, and extracted with DCM. The combined organic layers were dried over $\mathrm{Na}_{2} \mathrm{SO}_{4}$, concentrated, and purified by MPLC (hexanes:EtOAc = 7:3) to afford $(-)-\left[3 S-\left(3 R^{*}, 4 S^{*}, 5 S^{*}, 9 S^{*}\right), 6 E\right]-10-[[(1,1-$ dimethylethyl)dimethylsilyl]oxy]-3,9-bis-[(4-methoxyphenyl)methoxy]-2,4,7trimethyldeca-1,6-dien-5-ol (S30, $255 \mathrm{mg}, 34 \%$ ) and its C5-epimer (S30b, $265 \mathrm{mg}, 34 \%$ ), each as a colorless oil. S30: ${ }^{1} \mathrm{H}$ NMR $\left(500 \mathrm{MHz}, \mathrm{CDCl}_{3}\right) \delta 7.27(\mathrm{~d}, J=8.8 \mathrm{~Hz}, 2 \mathrm{H}), 7.24(\mathrm{~d}$, 
$J=8.7 \mathrm{~Hz}, 2 \mathrm{H}), 6.88(\mathrm{~d}, J=8.8 \mathrm{~Hz}, 2 \mathrm{H}), 6.84(\mathrm{~d}, J=8.8 \mathrm{~Hz}, 2 \mathrm{H}), 5.23($ broad d, $J=8.4 \mathrm{~Hz}$, $1 \mathrm{H}), 5.03(\mathrm{~s}, 1 \mathrm{H}), 5.00(\mathrm{~s}, 1 \mathrm{H}), 4.59(\mathrm{~d}, J=11.4 \mathrm{~Hz}, 1 \mathrm{H}), 4.52(\mathrm{~d}, J=11.2 \mathrm{~Hz}, 1 \mathrm{H}), 4.49(\mathrm{~d}$, $J=11.2 \mathrm{~Hz}, 1 \mathrm{H}), 4.29(\mathrm{ddd}, J=4.6,7.3$, and $9.0 \mathrm{~Hz}, 1 \mathrm{H}), 4.21(\mathrm{~d}, J=11.4 \mathrm{~Hz}, 1 \mathrm{H}), 4.00(\mathrm{~d}$, $J=4.0 \mathrm{~Hz}, 1 \mathrm{H}), 3.79(\mathrm{~s}, 3 \mathrm{H}), 3.77(\mathrm{~s}, 3 \mathrm{H}), 3.54-3.69(\mathrm{~m}, 3 \mathrm{H}), 2.25(\mathrm{dd}, J=4.3$ and 13.9 $\mathrm{Hz}, 1 \mathrm{H}), 2.18$ (dd, $J=7.7$ and $13.9 \mathrm{~Hz}, 1 \mathrm{H}), 2.18(\mathrm{~d}, J=4.6 \mathrm{~Hz}, 1 \mathrm{H}), 1.73$ (ddq, $J=4.3,7.2$ and $7.2 \mathrm{~Hz}, 1 \mathrm{H}), 1.70(\mathrm{~s}, 3 \mathrm{H}), 1.66(\mathrm{~s}, 3 \mathrm{H}), 0.90(\mathrm{~s}, 9 \mathrm{H}), 0.82(\mathrm{~d}, J=7.1 \mathrm{~Hz}, 3 \mathrm{H})$, and 0.05 (s, 6H). ${ }^{13} \mathrm{C}$ NMR $\left(125 \mathrm{MHz}, \mathrm{CDCl}_{3}\right) \delta 159.2,159.0,142.8,135.7,131.0,130.6,129.5$, 129.2, 113.8, 113.6, 112.5, 82.0, 78.1, 71.7, 70.4, 70.3, 65.6, 55.2, 42.1, 41.3, 25.9, 19.5, 18.3, 17.2, 10.7, -5.31, and -5.38. IR (neat) $3476,2952,2928,2856,1613,1514,1248$, 1090, 1037, and $836 \mathrm{~cm}^{-1}$. HRMS (FAB) Calcd for $\left(\mathrm{C}_{35} \mathrm{H}_{54} \mathrm{O}_{6} \mathrm{Si}+\mathrm{Na}\right)^{+}: 621.3582$, found: 621.3593. TLC $\mathrm{R}_{\mathrm{f}}=0.3$ in hexanes:EtOAc $4: 1[\alpha]^{\mathrm{RT}}-10.31^{\circ}\left(\mathrm{c}=0.38\right.$, DCM). S30b: ${ }^{1} \mathrm{H}$ $\operatorname{NMR}\left(500 \mathrm{MHz}, \mathrm{CDCl}_{3}\right) \delta 7.26(\mathrm{~d}, J=8.2 \mathrm{~Hz}, 2 \mathrm{H}), 7.25(\mathrm{~d}, J=9.0 \mathrm{~Hz}, 2 \mathrm{H}), 6.87(\mathrm{~d}, J=$ $8.2 \mathrm{~Hz}, 2 \mathrm{H}), 6.86(\mathrm{~d}, J=9.0 \mathrm{~Hz}, 2 \mathrm{H}), 5.37$ (broad d, $J=8.2 \mathrm{~Hz}, 1 \mathrm{H}), 5.07(\mathrm{~s}, 1 \mathrm{H}), 5.04(\mathrm{~s}$, $1 \mathrm{H}), 4.61(\mathrm{~d}, J=11.2 \mathrm{~Hz}, 1 \mathrm{H}), 4.50(\mathrm{~d}, J=11.2 \mathrm{~Hz}, 1 \mathrm{H}), 4.49(\mathrm{~d}, J=11.2 \mathrm{~Hz}, 1 \mathrm{H}), 4.44$ (ddd, $J=2.9,2.9$, and $8.2,1 \mathrm{H}), 4.19(\mathrm{~d}, J=11.2 \mathrm{~Hz}, 1 \mathrm{H}), 3.83(\mathrm{~d}, J=5.6 \mathrm{~Hz}, 1 \mathrm{H}), 3.81$ (s, $3 \mathrm{H}), 3.79(\mathrm{~s}, 3 \mathrm{H}), 3.53-3.66(\mathrm{~m}, 3 \mathrm{H}), 2.25(\mathrm{dd}, J=5.1$ and $13.9 \mathrm{~Hz}, 1 \mathrm{H}), 2.17(\mathrm{~d}, J=2.9$, $1 \mathrm{H}), 2.16(\mathrm{dd}, J=7.2$ and $13.9 \mathrm{~Hz}, 1 \mathrm{H}), 1.73(\mathrm{ddq}, J=3.0,5.6$, and $6.9 \mathrm{~Hz}, 1 \mathrm{H}), 1.70$ (s, $3 \mathrm{H}), 1.57(\mathrm{~s}, 3 \mathrm{H}), 0.97(\mathrm{~d}, J=7.3 \mathrm{~Hz}, 3 \mathrm{H}), 0.90(\mathrm{~s}, 9 \mathrm{H})$, and $0.05(\mathrm{~s}, 6 \mathrm{H}) \cdot{ }^{13} \mathrm{C}$ NMR $(125$ $\left.\mathrm{MHz}, \mathrm{CDCl}_{3}\right) \delta 159.1,159.0,142.6,134.8,131.0,130.5,129.4,129.3,129.2,113.8,113.7$, 113.4, 85.6, 78.4, 71.8, 70.7, 70.3, 65.6, 55.2, 41.9, 41.0, 25.9, 18.5, 18.3, 17.3, 8.0, -5.31, and -5.38. IR (neat) 3476, 2952, 2928, 2855, 1612, 1513, 1464, 1247, 1081, 1037, and 836 $\mathrm{cm}^{-1}$. TLC $\mathrm{R}_{\mathrm{f}}=0.3$ in hexanes:EtOAc 4:1. $[\alpha]^{\mathrm{RT}}-34.06^{\circ}(\mathrm{c}=0.31$, DCM $)$.

To alcohol S30 (300 mg, $0.502 \mathrm{mmol})$ in DMF ( $3 \mathrm{~mL})$ was added MeI (0.37 mL, 6.00 $\mathrm{mmol}$ ) and $\mathrm{NaH}$ ( $25 \%$ dispersion in mineral oil, $240 \mathrm{mg}, 2.51 \mathrm{mmol}$ ) at rt. The mixture was stirred for $24 \mathrm{~h}$, quenched with aqueous $\mathrm{NH}_{4} \mathrm{Cl}(10 \mathrm{~mL})$, diluted with $\mathrm{H}_{2} \mathrm{O}$, extracted with $\mathrm{Et}_{2} \mathrm{O}(3 \times 10 \mathrm{~mL})$, washed with $\mathrm{H}_{2} \mathrm{O}$, and dried over $\mathrm{Na}_{2} \mathrm{SO}_{4} . \mathrm{MPLC}$ (hexanes:EtOAc $=$ 19:1) separation gave $\mathbf{4 2}(245 \mathrm{mg}, 80 \%)$ as a colorless oil. ${ }^{1} \mathrm{H}$ NMR $\left(500 \mathrm{MHz}, \mathrm{CDCl}_{3}\right) \delta$ $7.27(\mathrm{~d}, J=8.6 \mathrm{~Hz}, 2 \mathrm{H}), 7.24(\mathrm{~d}, J=8.6 \mathrm{~Hz}, 2 \mathrm{H}), 6.88(\mathrm{~d}, J=8.6 \mathrm{~Hz}, 2 \mathrm{H}), 6.84(\mathrm{~d}, J=8.6$ $\mathrm{Hz}, 2 \mathrm{H}), 5.06$ (broad d, $J=9.7 \mathrm{~Hz}, 1 \mathrm{H}), 4.98($ app s, $2 \mathrm{H}), 4.60(\mathrm{~d}, J=11.8 \mathrm{~Hz}, 1 \mathrm{H}), 4.50$ (d, $J=11.8 \mathrm{~Hz}, 1 \mathrm{H}), 4.50(\mathrm{~d}, J=11.3 \mathrm{~Hz}, 1 \mathrm{H}), 4.19(\mathrm{~d}, J=11.3 \mathrm{~Hz}, 1 \mathrm{H}), 4.00(\mathrm{~d}, J=3.9 \mathrm{~Hz}$, $1 \mathrm{H}), 3.80(\mathrm{~s}, 3 \mathrm{H}), 3.79(\mathrm{dd}, J=8.4$ and $9.4 \mathrm{~Hz}, 1 \mathrm{H}), 3.78(\mathrm{~s}, 3 \mathrm{H}), 3.57-3.69(\mathrm{~m}, 3 \mathrm{H}), 3.07$ (s, 3H), 2.30 (dd, $J=4.6$ and $14.0 \mathrm{~Hz}, 1 \mathrm{H}), 2.23(\mathrm{dd}, J=6.7$ and $14.0 \mathrm{~Hz}, 1 \mathrm{H}), 1.73$ (ddq, $J$ $=3.9,8.4$, and $7.0 \mathrm{~Hz}, 1 \mathrm{H}), 1.68($ app s, $6 \mathrm{H}), 0.90(\mathrm{~s}, 9 \mathrm{H}), 0.75(\mathrm{~d}, J=7.1 \mathrm{~Hz}, 3 \mathrm{H})$, and 0.05 (s, 6H). ${ }^{13} \mathrm{C}$ NMR $\left(125 \mathrm{MHz}, \mathrm{CDCl}_{3}\right) \delta 159.0,158.9,143.5,137.8,131.5,131.0,129.3$, $129.2,126.8,113.65,113.62,111.6,81.2,78.3,77.8,71.8,70.7,65.6,55.5,55.3,55.2,42.2$, 40.4, 25.9, 19.3, 18.3, 17.5, 9.6, -5.32, and -5.39. IR (neat) 2952, 2928, 2856, 1613, 1513, $1463,1247,1082$, and $837 \mathrm{~cm}^{-1}$. HRMS (FAB) Calcd for $\left(\mathrm{C}_{36} \mathrm{H}_{56} \mathrm{O}_{6} \mathrm{Si}+\mathrm{Na}\right)^{+}: 635.3738$, found: 635.3772. $\mathrm{TLC} R f=0.5$, hexanes:EtOAc $=4: 1 .[\alpha]^{\mathrm{RT}}-3.00^{\circ}(\mathrm{c}=0.95, \mathrm{DCM})$.

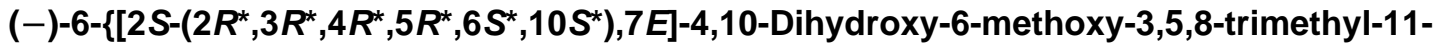 [[(1,1-dimethylethyl)dimethylsilyl]oxy]-2-[(trimethylsilyl)oxy]-undec-7-enyl\}-2,2- dimethyl-4H-1,3-dioxin-4-one (43)}

A flask containing $42(235 \mathrm{mg}, 0.384 \mathrm{mmol})$ was brought into a dry box where solid 9-BBN dimer (235 mg, $1.91 \mathrm{mmol}$ ) was added. The flask was taken out of the dry box, THF (4 mL) was added at $-78{ }^{\circ} \mathrm{C}$, and the solution was warmed to rt. The system was stirred for $18 \mathrm{~h}$ before $\mathrm{H}_{2} \mathrm{O}_{2}(30 \%, 0.6 \mathrm{~mL})$ and aqueous $\mathrm{NaOH}(10 \%, 3.8 \mathrm{~mL})$ were added at $0{ }^{\circ} \mathrm{C}$. After 45 min the reaction mixture was extracted with DCM and dried over $\mathrm{MgSO}_{4}$. Separation by flash chromatography (hexanes:EtOAc $=7: 3)$ gave a mixture of both diastereomers $(7: 1$ ratio). MPLC (hexanes:EtOAc $=7: 3)$ separation afforded the desired alcohol $(+)-\left[2 R-\left(2 R^{*}\right.\right.$, $\left.\left.3 R^{*}, 4 R^{*}, 5 R^{*}, 9 R^{*}\right), 6 E\right]-5$-methoxy-3,9-bis-[(4-methoxyphenyl)-methoxy]-2,4,7-

trimethyl-10-[[(1,1-dimethylethyl)dimethylsilyl]oxy]-deca-6-en-1-ol (S31, 168 mg, 70\%) 
and a minor amount of the $\mathrm{C} 2$-epimer (S32), each as a colorless oil. S31: ${ }^{1} \mathrm{H}$ NMR (500 $\left.\mathrm{MHz}, \mathrm{CDCl}_{3}\right) \delta 7.31(\mathrm{~d}, J=8.8 \mathrm{~Hz}, 2 \mathrm{H}), 7.25(\mathrm{~d}, J=8.7 \mathrm{~Hz}, 2 \mathrm{H}), 6.90(\mathrm{~d}, J=8.8 \mathrm{~Hz}, 2 \mathrm{H})$, $6.85(\mathrm{~d}, J=8.7 \mathrm{~Hz}, 2 \mathrm{H}), 5.01(\mathrm{dq}, J=9.1 \mathrm{and} 1.2 \mathrm{~Hz}, 1 \mathrm{H}), 4.62(\mathrm{~d}, J=11.3 \mathrm{~Hz}, 1 \mathrm{H}), 4.59$ $(\mathrm{d}, J=11.0 \mathrm{~Hz}, 1 \mathrm{H}), 4.56(\mathrm{~d}, J=11.0 \mathrm{~Hz}, 1 \mathrm{H}), 4.51(\mathrm{~d}, J=11.3 \mathrm{~Hz}, 1 \mathrm{H}), 3.85(\mathrm{dd}, J=1.8$ and $9.0 \mathrm{~Hz}, 1 \mathrm{H}), 3.81(\mathrm{~s}, 3 \mathrm{H}), 3.80(\mathrm{~s}, 3 \mathrm{H}), 3.75(\mathrm{dd}, J=9.7$ and $9.7 \mathrm{~Hz}, 1 \mathrm{H}) 3.50-3.71(\mathrm{~m}$, $5 \mathrm{H}), 3.17(\mathrm{~s}, 3 \mathrm{H}), 2.73(\mathrm{dd}, J=3.3$ and $8.7 \mathrm{~Hz}, 1 \mathrm{H}), 2.33(\mathrm{dd}, J=5.1$ and $14.0 \mathrm{~Hz}, 1 \mathrm{H}), 2.25$ (dd, $J=7.5$ and $14.0 \mathrm{~Hz}, 1 \mathrm{H}$ ), 1.92 (ddq, $J=4.0,9.0$, and $7.0 \mathrm{~Hz}, 1 \mathrm{H}$ ), 1.65 (ddq, $J=1.4$, 8.3, and $6.8 \mathrm{~Hz}, 1 \mathrm{H}), 1.68(\mathrm{~d}, J=1.5 \mathrm{~Hz}, 3 \mathrm{H}), 0.90(\mathrm{~s}, 9 \mathrm{H}), 0.83(\mathrm{~d}, J=7.1 \mathrm{~Hz}, 3 \mathrm{H}), 0.80(\mathrm{~d}$, $J=7.1 \mathrm{~Hz}, 3 \mathrm{H}), 0.061(\mathrm{~s}, 3 \mathrm{H})$, and $0.059(\mathrm{~s}, 3 \mathrm{H}) .{ }^{13} \mathrm{C} \mathrm{NMR}\left(125 \mathrm{MHz}, \mathrm{CDCl}_{3}\right) \delta 159.2$, 159.1, 138.2, 131.0, 130.9, 129.3, 129.3, 127.6, 113.9, 113.6, 81.8, 78.4, 78.2, 74.2, 71.8, $67.2,65.5,55.3,55.3,55.1,42.2,40.4,38.3,25.9,18.3,17.4,14.1,9.8,-5.33$, and -5.40 . IR (neat) 3470, 2953, 2928, 2857, 1613, 1513, 1248, 1090, 1036, and $837 \mathrm{~cm}^{-1}$. HRMS (FAB) Calcd for $\left(\mathrm{C}_{36} \mathrm{H}_{58} \mathrm{O}_{7} \mathrm{Si}+\mathrm{Na}\right)^{+}:$653.3844, found: 653.3851. TLC Rf $=0.2$, hexanes:EtOAc $=7: 3 .[\alpha]^{\mathrm{RT}}+23.2^{\circ}(\mathrm{c}=0.45, \mathrm{DCM}) . \mathbf{S 3 2}:{ }^{1} \mathrm{H}$ NMR $\left(500 \mathrm{MHz}, \mathrm{CDCl}_{3}\right) \delta$ $7.29(\mathrm{~d}, J=8.6 \mathrm{~Hz}, 2 \mathrm{H}), 7.25(\mathrm{~d}, J=8.6 \mathrm{~Hz}, 2 \mathrm{H}), 6.89(\mathrm{~d}, J=8.6 \mathrm{~Hz}, 2 \mathrm{H}), 6.85(\mathrm{~d}, J=8.6$ $\mathrm{Hz}, 2 \mathrm{H}), 5.01(\mathrm{~d}, J=9.8 \mathrm{~Hz}, 1 \mathrm{H}), 4.62(\mathrm{~d}, J=10.9 \mathrm{~Hz}, 1 \mathrm{H}), 4.58(\mathrm{~d}, J=10.4 \mathrm{~Hz}, 1 \mathrm{H}), 4.48$ $(\mathrm{d}, J=10.9 \mathrm{~Hz}, 1 \mathrm{H}), 4.51(\mathrm{~d}, J=10.3 \mathrm{~Hz}, 1 \mathrm{H}), 3.80(\mathrm{~s}, 3 \mathrm{H}), 3.79(\mathrm{~s}, 3 \mathrm{H}), 3.78(\mathrm{dd}, J=2.3$ and $7.0 \mathrm{~Hz}, 1 \mathrm{H}), 3.50-3.70(\mathrm{~m}, 6 \mathrm{H}), 3.12(\mathrm{~s}, 3 \mathrm{H}), 2.31(\mathrm{dd}, J=5.1$ and $14.2 \mathrm{~Hz}, 1 \mathrm{H}), 2.24$ (dd $J=7.2$ and $14.2 \mathrm{~Hz}, 1 \mathrm{H}), 1.96(\mathrm{ddq}, J=6.0,7.0$, and $7.0 \mathrm{~Hz}, 1 \mathrm{H}), 1.72(\mathrm{ddq}, J=2.1$, 6.6, and $9.0 \mathrm{~Hz}, 1 \mathrm{H}), 1.67(\mathrm{~d}, J=1.5 \mathrm{~Hz}, 3 \mathrm{H}), 1.01(\mathrm{~d}, J=7.3 \mathrm{~Hz}, 3 \mathrm{H}), 0.90(\mathrm{~s}, 9 \mathrm{H}), 0.85(\mathrm{~d}$, $J=6.9 \mathrm{~Hz}, 3 \mathrm{H}$ ), and 0.06 (s, 6H). IR (neat) 3434, 2953, 2928, 2856, 1613, 1513, 1247, 1096, and $837 \mathrm{~cm}^{-1}$. HRMS (FAB) Calcd for $\left(\mathrm{C}_{36} \mathrm{H}_{58} \mathrm{O}_{7} \mathrm{Si}+\mathrm{Na}\right)^{+}: 653.3844$, Found: 653.3857. TLC Rf $=0.2$, hexanes:EtOAc $=7: 3 .[\alpha]^{\mathrm{RT}}+6.62^{\circ}(\mathrm{c}=0.64, \mathrm{DCM})$.

Dess-Martin periodinane (158 $\mathrm{mg}, 0.37 \mathrm{mmol})$ was added to alcohol $\mathbf{S 3 1}(158 \mathrm{mg}, 0.25$ $\mathrm{mmol})$ and DCM $(12 \mathrm{~mL})$ at $\mathrm{rt}$. The resulting cloudy mixture was stirred $1 \mathrm{~h}$ before being placed directly onto a flash column. Flash chromatography (hexanes:EtOAc $=9: 1$ to 4:1)

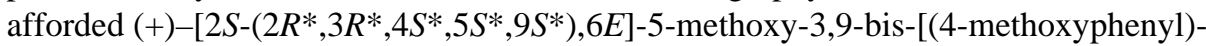
methoxy]-2,4,7-trimethyl-10-[[(1,1-dimethylethyl)dimethylsilyl]oxy]-6-decenal (S33, 119 $\mathrm{mg}, 76 \%)$ as a colorless oil. $\mathbf{S 3 3}{ }^{1} \mathrm{H}$ NMR $\left(500 \mathrm{MHz}, \mathrm{CDCl}_{3}\right) \delta 9.73(\mathrm{~d}, J=3.0 \mathrm{~Hz}, 1 \mathrm{H})$, $7.25(\mathrm{~d}, J=8.7 \mathrm{~Hz}, 2 \mathrm{H}), 7.24(\mathrm{~d}, J=8.7 \mathrm{~Hz}, 2 \mathrm{H}), 6.88(\mathrm{~d}, J=8.7 \mathrm{~Hz}, 2 \mathrm{H}), 6.85(\mathrm{~d}, J=8.7$ $\mathrm{Hz}, 2 \mathrm{H}), 5.02(\mathrm{~d}, J=9.6 \mathrm{~Hz}, 1 \mathrm{H}), 4.62(\mathrm{~d}, J=11.4 \mathrm{~Hz}, 1 \mathrm{H}), 4.51(\mathrm{~s}, 2 \mathrm{H}), 4.50(\mathrm{~d}, J=11.4$ $\mathrm{Hz}, 1 \mathrm{H}), 4.15(\mathrm{dd}, J=2.2$ and $9.3 \mathrm{~Hz}, 1 \mathrm{H}), 3.80(\mathrm{~s}, 3 \mathrm{H}), 3.79(\mathrm{~s}, 3 \mathrm{H}), 3.82(\mathrm{~m}, 1 \mathrm{H}), 3.57-$ $3.70(\mathrm{~m}, 3 \mathrm{H}), 3.17(\mathrm{~s}, 3 \mathrm{H}), 2.67(\mathrm{ddq}, J=3.0,9.3$, and $7.0 \mathrm{~Hz}, 1 \mathrm{H}), 2.33(\mathrm{dd}, J=5.0$ and $14.0 \mathrm{~Hz}, 1 \mathrm{H}), 2.25(\mathrm{dd}, J=7.4$ and $14.0 \mathrm{~Hz}, 1 \mathrm{H}), 1.68(\mathrm{~d}, J=1.4 \mathrm{~Hz}, 3 \mathrm{H}), 1.62(\mathrm{ddq}, J=$ $2.2,9.1$, and $7.0 \mathrm{~Hz}, 1 \mathrm{H}), 0.97(\mathrm{~d}, J=7.5 \mathrm{~Hz}, 3 \mathrm{H}), 0.91(\mathrm{~s}, 9 \mathrm{H}), 0.80(\mathrm{~d}, J=7.5 \mathrm{~Hz}, 3 \mathrm{H})$, $0.061(\mathrm{~s}, 3 \mathrm{H})$, and $0.059(\mathrm{~s}, 3 \mathrm{H}) .{ }^{13} \mathrm{C}$ NMR $\left(125 \mathrm{MHz}, \mathrm{CDCl}_{3}\right) \delta 205.1,159.1,159.2,138.5$, $130.9,130.9,129.3,129.2,127.4,113.8,113.7,78.4,78.1,77.7,73.9,71.8,65.5,55.28$, 55.27, 55.0, 49.8, 42.2, 40.5, 25.9, 18.3, 17.4, 11.1, 9.5, -5.32, and -5.39. IR (neat) 2952, 2932, 2856, 1724, 1613, 1513, 1248,1036, and $837 \mathrm{~cm}^{-1}$. Anal. Calcd for $\mathrm{C}_{36} \mathrm{H}_{56} \mathrm{O}_{7} \mathrm{Si}: \mathrm{C}$, 68.75; H: 8.97. found: C, 69.14; H, 8.65. TLC Rf $=0.4$, hexanes:EtOAc $=4: 1 .[\alpha]^{\mathrm{RT}}+2.0^{\circ}$ $(\mathrm{c}=0.84, \mathrm{DCM})$.

$\mathrm{BF}_{3} \cdot \mathrm{Et}_{2} \mathrm{O}$ (45 $\left.\mu \mathrm{L}, 0.36 \mathrm{mmol}\right)$ was added over $2 \mathrm{~min}$ to aldehyde $\mathbf{S 3 3}$ (113 $\mathrm{mg}, 0.18 \mathrm{mmol}$ ) and ketene acetal $20(385 \mathrm{mg}, 1.8 \mathrm{mmol})$ in DCM $(9 \mathrm{~mL})$ at $-78{ }^{\circ} \mathrm{C}$. After $45 \mathrm{~min}$ the reaction mixture was allowed to warm to room temperature before being quenched with saturated aqueous $\mathrm{NaHCO}_{3}$. The resulting mixture was extracted with DCM, dried over $\mathrm{MgSO}_{4}$, and concentrated. Flash chromatography (hexanes:EtOAc $=7: 3$ to 1:1) gave (+)-6$\left\{\left[2 S-\left(2 R^{*}, 3 S^{*}, 4 S^{*}, 5 S^{*}, 6 S^{*}, 10 S^{*}\right), 7 E\right]-2\right.$-hydroxy-6-methoxy-5,10-bis-[(4-

methoxyphenyl)methoxy]-3,5,8-trimethyl-11-[[(1,1-dimethylethyl)dimethylsilyl]-oxy]undec-7-enyl \}-2,2-dimethyl-4H-1,3-dioxin-4-one (S34, $125 \mathrm{mg}, 90 \%)$. S34 ${ }^{1} \mathrm{H}$ NMR (500 $\left.\mathrm{MHz}, \mathrm{CDCl}_{3}\right) \delta 7.29(\mathrm{~d}, J=8.4 \mathrm{~Hz}, 2 \mathrm{H}), 7.25(\mathrm{~d}, J=8.4 \mathrm{~Hz}, 2 \mathrm{H}), 6.90(\mathrm{~d}, J=8.4 \mathrm{~Hz}, 2 \mathrm{H})$, $6.85(\mathrm{~d}, J=8.4 \mathrm{~Hz}, 2 \mathrm{H}), 5.27(\mathrm{~s}, 1 \mathrm{H}), 5.02(\mathrm{~d}, J=9.6 \mathrm{~Hz}, 1 \mathrm{H}), 4.63(\mathrm{~d}, J=10.6 \mathrm{~Hz}, 1 \mathrm{H})$, 
$4.61(\mathrm{~d}, J=10.6 \mathrm{~Hz}, 1 \mathrm{H}), 4.51(\mathrm{~d}, J=10.6 \mathrm{~Hz}, 1 \mathrm{H}), 4.50(\mathrm{~d}, J=10.6 \mathrm{~Hz}, 1 \mathrm{H}), 4.16(\mathrm{~m}, 1 \mathrm{H})$, $3.87(\mathrm{dd}, J=2.4$ and $8.2 \mathrm{~Hz}, 1 \mathrm{H}), 3.81(\mathrm{~s}, 3 \mathrm{H}), 3.79(\mathrm{~s}, 3 \mathrm{H}), 3.78(\mathrm{dd}, J=9.6$ and $9.6 \mathrm{~Hz}$, $1 \mathrm{H}), 3.58-3.72(\mathrm{~m}, 3 \mathrm{H}), 3.20(\mathrm{~s}, 3 \mathrm{H}), 2.38(\mathrm{dd}, J=9.45$ and $14.4 \mathrm{~Hz}, 2 \mathrm{H}), 2.35(\mathrm{dd}, J=4.53$ and $12.8 \mathrm{~Hz}, 1 \mathrm{H}), 2.33(\mathrm{~d}, J=5.8 \mathrm{~Hz}, 1 \mathrm{H}), 2.26(\mathrm{dd}, J=6.8$ and $12.8 \mathrm{~Hz}, 1 \mathrm{H}), 2.23(\mathrm{dd}, J=$ 4.15 and $14.4 \mathrm{~Hz}, 1 \mathrm{H}), 1.70(\mathrm{~m}, 2 \mathrm{H}), 1.69(\mathrm{~s}, 3 \mathrm{H}), 0.91(\mathrm{~s}, 9 \mathrm{H}), 0.85(\mathrm{~d}, J=7.0 \mathrm{~Hz}, 3 \mathrm{H})$, $0.81(\mathrm{~d}, J=7.0 \mathrm{~Hz}, 3 \mathrm{H})$, and $0.06(\mathrm{~s}, 6 \mathrm{H}) .{ }^{13} \mathrm{C}$ NMR $\left(125 \mathrm{MHz}, \mathrm{CDCl}_{3}\right) \delta 169.9,161.1$, 159.3, 159.0, 138.3, 130.8, 130.7, 129.4, 129.3, 127.3, 113.8, 113.6, 106.4, 94.7, 79.9, 78.4, 78.1, 74.2, 71.7, 68.7, 65.4, 55.2, 55.2, 55.0, 42.1, 40.8, 40.7, 39.3, 25.9, 25.2, 24.7, 18.2, 17.4, 10.6, 9.8, -5.37, and -5.43. IR (neat) 3457, 2928, 2856, 1726, 1634, 1613, 1512, 1389 , and $1375 \mathrm{~cm}^{-1}$. HRMS (FAB) Calcd for $\left(\mathrm{C}_{43} \mathrm{H}_{66} \mathrm{O}_{10} \mathrm{Si}+\mathrm{Na}\right)^{+}: 793.4318$, found: 793.4336. $\mathrm{TLC} \mathrm{Rf}=0.35$, hexanes:EtOAc $=1: 1 .[\alpha]^{\mathrm{RT}}+7.2^{\circ}(\mathrm{c}=0.31, \mathrm{DCM})$.

TMSCl (0.046 mL, $0.36 \mathrm{mmol})$ was added to a solution of $\mathbf{S 3 4}(28 \mathrm{mg}, 0.036 \mathrm{mmol}), \mathrm{Et}_{3} \mathrm{~N}$ $(0.15 \mathrm{~mL}, 1.08 \mathrm{mmol})$, and DCM $(0.3 \mathrm{~mL})$ at rt. The resulting mixture was stirred until the reaction was deemed complete by TLC, ca. $4 \mathrm{~h}$, at which time the solution had taken on a pink color. The mixture was quenched with aqueous $\mathrm{NaHCO}_{3}$, extracted with DCM, dried over $\mathrm{Na}_{2} \mathrm{SO}_{4}$, and concentrated to furnish crude silyl ether, which was carried forward without purification. This oil was first dissolved in wet DCM $(1.2 \mathrm{~mL})$ and $\mathrm{H}_{2} \mathrm{O}(100 \mu \mathrm{L})$. DDQ ( $65 \mathrm{mg}, 0.29 \mathrm{mmol}$ ) was added at $\mathrm{rt}$, and the mixture was stirred for $0.5 \mathrm{~h}$. Aqueous $\mathrm{NaHCO}_{3}$ was added, the mixture was stirred for 5 min, diluted with water, and extracted with DCM. The organic layers were dried over $\mathrm{Na}_{2} \mathrm{SO}_{4}$ and concentrated. Flash chromatography (hexanes:EtOAc $=7: 3$ to $100 \%$ EtOAc) furnished diol $\mathbf{4 3}(17 \mathrm{mg}, 78 \%)$ as a colorless oil. $43{ }^{1} \mathrm{H}$ NMR $\left(500 \mathrm{MHz}, \mathrm{CDCl}_{3}\right) \delta 5.29(\mathrm{~s}, 1 \mathrm{H}), 5.20(\mathrm{~d}, J=9.3 \mathrm{~Hz}, 1 \mathrm{H}), 4.36$ (ddd, $J=2.2,4.3,8.6 \mathrm{~Hz}, 1 \mathrm{H}), 3.95(\mathrm{dd}, J=6.7$ and $9.4 \mathrm{~Hz}, 1 \mathrm{H}), 3.83$ (ddd, $J=1.5,1.5$, and $9.7 \mathrm{~Hz}, 1 \mathrm{H}), 3.81(\mathrm{~m}, 1 \mathrm{H}), 3.63(\mathrm{dd}, J=3.6,9.9 \mathrm{~Hz}, 1 \mathrm{H}), 3.46(\mathrm{dd}, J=6.8$ and $9.5 \mathrm{~Hz}, 1 \mathrm{H})$, $3.44(\mathrm{~d}, J=1.8 \mathrm{~Hz}, 1 \mathrm{H}), 3.25(\mathrm{~s}, 3 \mathrm{H}), 2.47(\mathrm{dd}, J=8.8$ and $13.8 \mathrm{~Hz}, 2 \mathrm{H}), 2.41(\mathrm{~d}, J=3.6$, $1 \mathrm{H}), 2.31(\mathrm{dd}, J=4.3$ and $13.8 \mathrm{~Hz}, 1 \mathrm{H}), 2.21(\mathrm{bs}, 1 \mathrm{H}), 2.19(\mathrm{bs}, 1 \mathrm{H}), 1.75(\mathrm{~d}, J=1.4 \mathrm{~Hz}$, $3 \mathrm{H}), 1.70(\mathrm{~s}, 3 \mathrm{H}), 1.68(\mathrm{~s}, 3 \mathrm{H}), 1.63(\mathrm{~m}, 2 \mathrm{H}) 0.91(\mathrm{~s}, 9 \mathrm{H}), 0.86(\mathrm{~d}, J=7.2 \mathrm{~Hz}, 3 \mathrm{H}), 0.74(\mathrm{~d}, J$ $=6.8 \mathrm{~Hz}, 3 \mathrm{H}), 0.12(\mathrm{~s}, 9 \mathrm{H})$, and $0.07(\mathrm{~s}, 6 \mathrm{H}) .{ }^{13} \mathrm{C} \mathrm{NMR}\left(125 \mathrm{MHz}, \mathrm{CDCl}_{3}\right) \delta 169.6,161.1$, 137.0, 127.6, 106.4, 95.2, 80.2, 70.9, 70.9, 69.7, 66.9, 56.1, 43.6, 40.6, 39.6, 39.2, 26.2, 25.8, 24.0, 18.3, 17.0, 10.6, 8.4, 0.19, -5.38, and -5.41 . IR (neat) 3502, 2953, 2931, 2857, $1734,1635,1464,1390,1375,1273,1251,1085$ and $840 \mathrm{~cm}^{-1}$. HRMS (FAB) Calcd for $\left(\mathrm{C}_{30} \mathrm{H}_{58} \mathrm{O}_{8} \mathrm{Si}_{2}+\mathrm{Na}\right)^{+}:$625.3562, found: 625.3570. TLC Rf $=0.6$, hexanes:EtOAc $=1: 1$. $[\alpha]^{\mathrm{RT}}-22.8^{\circ}(\mathrm{c}=0.29, \mathrm{DCM})$.

\section{$(-)-\left[1 S^{\star}\left(1 R^{\star}, 5 S^{\star}, 9 S^{\star}, 10 S^{\star}, 11 R^{\star}, 12 R^{\star}, 13 R^{\star}\right) 7 E\right)$-1-Hydroxy-9-methoxy-7,10,12-trimethyl-5- [[[(1,1-dimethylethyl)dimethylsilyl]oxy]methyl]-13-[(trimethylsilyl)oxy]-4,15- dioxabicyclo[9.3.1]pentadec-7-en-3-one (44)}

Benzene $(200 \mathrm{~mL})$ was placed in a round bottom flask equipped with a Dean-Stark trap. The flask was heated and $40 \mathrm{~mL}$ of benzene was removed through the trap. The condenser was removed and diol $43(16 \mathrm{mg}, 0.027 \mathrm{mmol})$ in benzene $(2 \mathrm{~mL})$ was transferred into the flask. The condenser was replaced and the mixture was refluxed for $12 \mathrm{~h}$. The reaction mixture was cooled to rt, concentrated, and purified by flash chromatography (hexanes:EtOAc $=9: 1$ ) to afford macrolactone $44(11 \mathrm{mg}, 78 \%) .{ }^{1} \mathrm{H}$ NMR $\left(500 \mathrm{MHz}, \mathrm{CDCl}_{3}\right) \delta 5.37$ (dddd, $J=$ $11.6,6.8,4.4,2.5 \mathrm{~Hz}, 1 \mathrm{H}), 5.28(\mathrm{dq}, J=9.5,1.2 \mathrm{~Hz}, 1 \mathrm{H}), 5.07(\mathrm{~d}, J=2.5 \mathrm{~Hz}, 1 \mathrm{H}), 3.80(\mathrm{dd}$, $J=9.3,2.4 \mathrm{~Hz}, 1 \mathrm{H}), 3.77(\mathrm{~m}, 1 \mathrm{H}), 3.70(\mathrm{dd}, J=11.0,6.7 \mathrm{~Hz}, 1 \mathrm{H}), 3.66(\mathrm{dd}, J=11.1,4.5$ $\mathrm{Hz}, 1 \mathrm{H}), 3.61$ (dd, $J=10.4,2.7 \mathrm{~Hz}, 1 \mathrm{H}), 3.24(\mathrm{~s}, 3 \mathrm{H}), 2.50(\mathrm{~d}, J=12.8 \mathrm{~Hz}, 1 \mathrm{H}), 2.40(\mathrm{~d}, J=$ $12.8 \mathrm{~Hz}, 1 \mathrm{H}), 2.24(\mathrm{dd}, J=13.4,2.6 \mathrm{~Hz}, 1 \mathrm{H}), 2.20(\mathrm{ddq}, J=2.4,2.4,7.0 \mathrm{~Hz}, 2 \mathrm{H}), 2.16(\mathrm{dd}$, $J=13.4,11.7 \mathrm{~Hz}, 1 \mathrm{H}), 1.97(\mathrm{dd}, J=12.0,4.7 \mathrm{~Hz}, 1 \mathrm{H}), 1.72(\mathrm{~s}, 3 \mathrm{H}), 1.42(\mathrm{ddq}, J=10.2$, $10.2,6.1 \mathrm{~Hz}, 1 \mathrm{H}), 1.35$ (ddd, $J=11.9,10.7,2.4 \mathrm{~Hz}, 1 \mathrm{H}), 0.97(\mathrm{~d}, J=7.1 \mathrm{~Hz}, 3 \mathrm{H}), 0.89$ (d, $J$ $=6.7 \mathrm{~Hz}, 3 \mathrm{H}), 0.88(\mathrm{~s}, 9 \mathrm{H}), 0.13(\mathrm{~s}, 9 \mathrm{H}), 0.05(\mathrm{~s}, 3 \mathrm{H})$, and $0.05(\mathrm{~s}, 3 \mathrm{H}) .{ }^{13} \mathrm{C} \mathrm{NMR}(125$ 
$\left.\mathrm{MHz}, \mathrm{CDCl}_{3}\right) \delta 172.4,132.6,127.3,95.4,79.9,72.7,70.1,65.17,65.12,55.2,44.6,44.4$, $42.6,40.1,37.1,25.7,18.2,16.1,12.3,6.6,-0.02,-5.30$, and -5.33 . IR (neat) 3451,2956 , $2929,2859,1706,1251,1075$, and $838 \mathrm{~cm}^{-1}$. HRMS (FAB) calcd for $\left(\mathrm{C}_{27} \mathrm{H}_{52} \mathrm{O}_{7} \mathrm{Si}_{2}+\right.$ $\mathrm{Na})^{+}:$567.3144, found: 567.3159. TLC $\mathrm{R}_{\mathrm{f}}=0.4$, hexanes:EtOAc $=9: 1 .[\alpha]^{\mathrm{rt}}-19.8^{\circ}(\mathrm{c}=$ $0.93, \mathrm{DCM})$.

\section{(-)-[1 $\left.S^{\star}\left(1 R^{\star}, 5 S^{\star}, 9 S^{\star}, 10 S^{\star}, 11 R^{\star}, 12 R^{\star}, 13 R^{\star}\right) 7 E\right]-1-H y d r o x y-5-($ hydroxymethyl)-9- methoxy-7,10,12-trimethyl-13-[(trimethylsilyl)oxy]-4,15-dioxabicyclo[9.3.1]-pentadec-7- en-3-one (48)}

TBAF (0.28 mL, 1.0 M in THF, $0.28 \mathrm{mmol})$ was added to $44(11 \mathrm{mg}, 0.020 \mathrm{mmol})$ in THF $(0.5 \mathrm{~mL})$ at $\mathrm{rt}$ and stirred for $10 \mathrm{~min}$. The solution was placed on a pipette column and purified by flash chromatography (100\% EtOAc) to afford a diol that was immediately carried into the next reaction. TMSCl $(0.05 \mathrm{~mL}, 0.40 \mathrm{mmol})$ was added to a mixture of the diol, NEt3 $(0.17 \mathrm{~mL}, 1.21 \mathrm{mmol})$, and DCM $(0.2 \mathrm{~mL})$ at rt. The mixture was stirred for $2 \mathrm{~h}$, quenched with aqueous $\mathrm{NaHCO}_{3}$, diluted with water, extracted with DCM, dried over $\mathrm{Na}_{2} \mathrm{SO}_{4}$, and concentrated to give a crude oil. This lactone was dissolved in $\mathrm{MeOH}(1 \mathrm{~mL})$, $\mathrm{NaHCO}_{3}(5 \mathrm{mg})$ was added, and the resulting mixture was stirred for $2 \mathrm{~h}$. The mixture was diluted with water, extracted with DCM, dried over $\mathrm{Na}_{2} \mathrm{SO}_{4}$, and concentrated. Purification by flash chromatography (hexanes:EtOAc $=19: 1)$ afforded $\mathbf{4 8}(6 \mathrm{mg}, 70 \%$ over three steps). ${ }^{1} \mathrm{H}$ NMR $\left(500 \mathrm{MHz}, \mathrm{CDCl}_{3}\right) \delta 5.42$ (dddd, $J=2.4,3.7,6.2$, and $\left.8.7 \mathrm{~Hz}, 1 \mathrm{H}\right), 5.31(\mathrm{~d}$, $J=9.4 \mathrm{~Hz}, 1 \mathrm{H}), 5.00(\mathrm{~d}, J=2.4 \mathrm{~Hz}, 1 \mathrm{H}), 3.8(\mathrm{dd}, J=2.2$ and $9.6 \mathrm{~Hz}, 1 \mathrm{H}), 3.76(\mathrm{~m}, 3 \mathrm{H})$, $3.61(\mathrm{dd}, J=2.5,10.3 \mathrm{~Hz}, 1 \mathrm{H}), 3.24(\mathrm{~s}, 3 \mathrm{H}), 2.54(\mathrm{~d}, J=13.0 \mathrm{~Hz}, 1 \mathrm{H}), 2.44(\mathrm{~d}, J=13.0 \mathrm{~Hz}$, $1 \mathrm{H}), 2.30(\mathrm{dd}, J=12.0$ and $12.0 \mathrm{~Hz}, 1 \mathrm{H}), 2.21(\mathrm{~m}, 2 \mathrm{H}), 1.98(\mathrm{dd}, J=4.6$ and $12.0 \mathrm{~Hz}, 1 \mathrm{H})$, $1.74(\mathrm{~s}, 3 \mathrm{H}), 1.61(\mathrm{dd}, J=5.9$ and $5.9 \mathrm{~Hz}, 1 \mathrm{H}), 1.43(\mathrm{ddq}, J=10.3,10.3$, and $6.5 \mathrm{~Hz}, 1 \mathrm{H})$, 1.36 (ddd, $J=2.4,10.7$, and $12.0 \mathrm{~Hz}, 1 \mathrm{H}), 0.97(\mathrm{~d}, J=7.3 \mathrm{~Hz}, 3 \mathrm{H}), 0.90(\mathrm{~d}, J=6.4 \mathrm{~Hz}, 3 \mathrm{H})$, 0.14 (s, 9H). ${ }^{13} \mathrm{C}$ NMR $\left(125 \mathrm{MHz}, \mathrm{CDCl}_{3}\right) \delta 172.4,132.2,127.7,99.4,79.8,75.1,72.8$, 70.0, 64.9, 55.2, 44.7, 44.3, 42.4, 40.1, 37.0, 16.2, 12.4, 6.6, and 0.30. IR (neat) 3434, 2962, 2930, 2874, 1704, 1251, 1227, 1182, 1075, 891, and $841 \mathrm{~cm}^{-1}$. HRMS (FAB) Calcd for $\left(\mathrm{C}_{7} \mathrm{H}_{38} \mathrm{O}_{7} \mathrm{Si}+\mathrm{Na}\right)^{+}: 453.2279$, found: 453.2291. $\mathrm{TLC} \mathrm{R}_{\mathrm{f}}=0.5$, hexanes:EtOAc $=1: 1 .[\alpha]^{\mathrm{RT}}$ $-23.9^{\circ}(\mathrm{c}=0.33, \mathrm{DCM})$.

\section{$(-)-\left[1 S\left(1 R^{\star}, 5 S^{\star}, 9 S^{\star}, 10 S^{\star}, 11 S^{\star}, 12 S^{\star}, 13 R^{\star}\right) 7 E\right)-5-\left[(1 E, 3 E)-6-\left(\left(1 R^{\star}, 2 S^{\star}\right)-2-\right.\right.$ Chlorocyclopropyl)-1,3-hexadien-5-yn-1-yl]-1,13-dihydroxy-9-methoxy-7,10,12- trimethyl-4-15-dioxabicyclo[9.3.1]pentadec-7-en-3-one (49) 2,3a,3c,3d,3e}

Sulfur trioxide $•$ pyridine $(19 \mathrm{mg}, 0.12 \mathrm{mmol})$ was added to a mixture of alcohol $48(5 \mathrm{mg}$, $0.01 \mathrm{mmol})$, TEA $(24.0 \mu \mathrm{L}, 0.174 \mathrm{mmol})$, DCM $(100 \mu \mathrm{L})$, and DMSO $(300 \mu \mathrm{L})$ at $0{ }^{\circ} \mathrm{C}$. The mixture was stirred $0.5 \mathrm{~h}$ at which time it was diluted with EtOAc $(500 \mu \mathrm{L})$, quenched with aqueous $\mathrm{NH}_{4} \mathrm{Cl}$, and diluted with $\mathrm{H}_{2} \mathrm{O}(5 \mathrm{~mL})$. The aqueous layer was extracted with EtOAc $(3 \times 5 \mathrm{~mL})$. The combined organic layers were washed with brine $(5 \mathrm{~mL})$, washed with water $(5 \mathrm{~mL})$, dried over $\mathrm{Na}_{2} \mathrm{SO}_{4}$, and concentrated. The crude product was passed through a plug of silica gel (hexanes:EtOAc $=1: 1$ ) to afford crude aldehyde, which was immediately carried on to the next reaction. A solution of $\mathrm{n}-\mathrm{BuLi}(0.3 \mathrm{~mL}, 2.05 \mathrm{M}, 0.67 \mathrm{mmol})$ was added to neat hexamethyldisilazane $(0.14 \mathrm{~mL}, 0.67 \mathrm{mmol})$ in a round bottom flask at $0{ }^{\circ} \mathrm{C}$. The solution was stirred for $0.5 \mathrm{~h}$ at which time a white precipitate had formed. THF (2.9 $\mathrm{mL}$ ) was then added to make a $0.2 \mathrm{M}$ solution of LiHMDS. Phosphonate $\mathbf{4 3}^{3 \mathrm{c}, 3 \mathrm{e}}(14 \mathrm{mg}$, $0.051 \mathrm{mmol})$ was placed in a vial and dissolved in THF $(0.3 \mathrm{~mL})$. To this solution was added LiHMDS $(255 \mu \mathrm{L}, 0.2 \mathrm{M}$ in THF, $0.051 \mathrm{mmol})$ at $-78^{\circ} \mathrm{C}$, resulting in a dark brown color. The mixture was stirred $3 \mathrm{~min}$ at $-78{ }^{\circ} \mathrm{C}$ before the aldehyde from the previous step (in THF, $200 \mu \mathrm{L}$ ) was added. The vessel was immediately removed from the dry ice bath and allowed to warm to rt over 4 min before being quenched with aqueous $\mathrm{NH}_{4} \mathrm{Cl}$, extracted with EtOAc, dried over $\mathrm{Na}_{2} \mathrm{SO}_{4}$, and concentrated. The crude product was passed through a pipette column of silica gel (hexanes:EtOAc $=4: 1$ ) to afford the TMS protected product. 
The silylated product was dissolved in THF ( $0.5 \mathrm{~mL})$, TBAF (30 $\mu \mathrm{L}, 1.0 \mathrm{M}$ in THF) was added, and the resulting mixture was stirred for $5 \mathrm{~min}$ at rt. The solvent was removed with a stream of $\mathrm{N}_{2}$ and the resulting oil was loaded onto a flash column with DCM. Flash chromatography (hexanes:EtOAc $=7: 3$ to $1: 1)$ afforded $49(3 \mathrm{mg}, 54 \%) .{ }^{1} \mathrm{H}$ NMR $(500$ $\left.\mathrm{MHz}, \mathrm{CDCl}_{3}\right) \delta 6.48(\mathrm{dd}, J=10.9$ and $15.4 \mathrm{~Hz}, 1 \mathrm{H}), 6.27(\mathrm{dd}, J=10.9$ and $15.1 \mathrm{~Hz}, 1 \mathrm{H})$, $5.83(\mathrm{~m}, 1 \mathrm{H}), 5.76(\mathrm{dd}, J=6.2$ and $15.1 \mathrm{~Hz}, 1 \mathrm{H}), 5.57(\mathrm{dd}, J=2.2$ and $15.5 \mathrm{~Hz}, 1 \mathrm{H}), 5.31$ $(\mathrm{d}, J=9.7 \mathrm{~Hz}, 1 \mathrm{H}), 5.03(\mathrm{~d}, J=2.4 \mathrm{~Hz}, 1 \mathrm{H}), 3.80(\mathrm{dd}, J=2.4$ and $9.5 \mathrm{~Hz}, 1 \mathrm{H}), 3.79(\mathrm{~m}$, $1 \mathrm{H}), 3.61(\mathrm{dd}, J=2.6$ and $10.2 \mathrm{~Hz}, 1 \mathrm{H}), 3.24(\mathrm{~s}, 3 \mathrm{H}), 3.18(\mathrm{ddd}, J=3.2,5.6$, and $6.2 \mathrm{~Hz}$, $1 \mathrm{H}), 2.55(\mathrm{~d}, J=12.9 \mathrm{~Hz}, 1 \mathrm{H}), 2.44(\mathrm{~d}, J=12.9 \mathrm{~Hz}, 1 \mathrm{H}), 2.29$ (m, 2H), 2.22 (ddq, $J=2.5$, 2.5, and $6.9 \mathrm{~Hz}, 1 \mathrm{H}), 2.10(\mathrm{dd}, J=4.6$ and $11.8 \mathrm{~Hz}, 1 \mathrm{H}), 1.78(\mathrm{~m}, 1 \mathrm{H}), 1.74(\mathrm{~s}, 3 \mathrm{H}), 1.39$ $(\mathrm{m}, 1 \mathrm{H}) 1.30(\mathrm{~m}, 4 \mathrm{H})$, and $0.98(\operatorname{app~d}, J=6.8 \mathrm{~Hz}, 6 \mathrm{H}) .{ }^{13} \mathrm{C} \mathrm{NMR}\left(125 \mathrm{MHz}, \mathrm{CDCl}_{3}\right) \delta$ $171.8,140.1,132.7,132.3,130.9,127.8,112.5,95.3,92.2,79.8,77.2,75.0,71.5,69.5,55.2$, $46.9,44.7,43.7,40.1,36.8,34.3,19.3,16.1,12.06,12.03$, and 6.5. IR (neat) 3452, 3364, 2953, 2923, 2853, 1700, 1465, 1457, and $1225 \mathrm{~cm}^{-1}$. HRMS (FAB) Calcd for $\left(\mathrm{C}_{26} \mathrm{H}_{35} \mathrm{ClO}_{6}\right.$ $+\mathrm{Na})^{+}: 501.2014$, found: 501.2037. TLC $\mathrm{R}_{\mathrm{f}}=0.5$, hexanes:EtOAc $=1: 1 .[\alpha]^{\mathrm{RT}}-53.8^{\circ}(\mathrm{c}=$ $\left.0.5, \mathrm{CHCl}_{3}\right)$.

\section{$(-)-\left\{3 a R\left(3 a R^{\star}, 4 S^{\star}, 6 R, 7 R, 7 a R^{\star}\right)\right\}-6-\left[\left[\left(1 S^{\star}, 5 R^{\star}, 7 E, 9 R^{\star}, 10 R^{\star}, 11 R^{\star}, 12 R^{\star}, 13 S^{\star}\right)-5-\left[(1 E, 3 E)-6-\left[\left(1 S^{\star}\right.\right.\right.\right.\right.$, $\left.2 R^{\star}\right)$-2-Chlorocyclopropyl]-1,3-hexadien-5-yn-1-yl]-1-hydroxy-9-methoxy-7,10,12- trimethyl-3-oxo-4,15-dioxabicyclo[9.3.1]pentadec-7-en-13-yl]oxy]-7-methoxy-4,7a- dimethyl-4H-pyrano[3,4-d]oxazol-2(3H)-one (1)}

To a mixture of the aglycon 49 ( $2 \mathrm{mg}, 0.004 \mathrm{mmol})$, carbohydrate $34^{3 \mathrm{f}}(3 \mathrm{mg}, 0.008)$, and powdered molecular sieves $(20 \mathrm{mg})$ in DCM $(300 \mu \mathrm{L})$ was added TMSOTf $(0.055 \mathrm{M}$ in DCM, $15 \mu \mathrm{L}, 0.836 \mu \mathrm{mol}$ ) at $0{ }^{\circ} \mathrm{C}$. TLC still showed starting material after stirring for 30 min and so additional carbohydrate $\mathbf{3}(1.5 \mathrm{mg}, 0.004 \mathrm{mmol})$ was added. After the addition, TLC showed absence of starting material within $10 \mathrm{~min}$. The mixture was quenched with saturated aqueous $\mathrm{NaHCO}_{3}$. The resulting solution was diluted with $\mathrm{H}_{2} \mathrm{O}$, extracted with DCM, dried over $\mathrm{Na}_{2} \mathrm{SO}_{4}$, and purified by HPLC (100\% EtOAc) to afford (-)-callipeltoside A (1) $(2 \mathrm{mg}, 70 \%) .{ }^{1} \mathrm{H}$ NMR $\left(500 \mathrm{MHz}, \mathrm{CD}_{3} \mathrm{OD}\right) \delta 6.49(\mathrm{dd}, J=10.8$ and $15.4 \mathrm{~Hz}, 1 \mathrm{H})$, $6.33(\mathrm{dd}, J=10.8$ and $14.1 \mathrm{~Hz}, 1 \mathrm{H}), 5.83(\mathrm{~m}, 1 \mathrm{H}), 5.80(\mathrm{~m}, 1 \mathrm{H}), 5.64(\mathrm{dd}, J=1.9$ and 15.4 $\mathrm{Hz}, 1 \mathrm{H}), 5.26(\mathrm{~d}, J=9.7 \mathrm{~Hz}, 1 \mathrm{H}), 4.71(\mathrm{~d}, J=6.2 \mathrm{~Hz}, 1 \mathrm{H}), 3.95(\mathrm{dd}, J=1.9$ and $6.5 \mathrm{~Hz}$, $1 \mathrm{H}), 3.87(\mathrm{dd}, J=2.4$ and $9.5 \mathrm{~Hz}, 1 \mathrm{H}), 3.72(\mathrm{dd}, J=4.7,10.7$, and $10.7 \mathrm{~Hz}, 1 \mathrm{H}), 3.64(\mathrm{dd}, J$ $=2.4$ and $10.4 \mathrm{~Hz}, 1 \mathrm{H}), 3.59(\mathrm{~s}, 3 \mathrm{H}), 3.44(\mathrm{~d}, J=1.9 \mathrm{~Hz}, 1 \mathrm{H}), 3.42(\mathrm{~d}, J=6.1 \mathrm{~Hz}, 1 \mathrm{H}), 3.24$ (m), $3.21(\mathrm{~s}, 3 \mathrm{H}), 2.52(\mathrm{~d}, J=12.8 \mathrm{~Hz}, 1 \mathrm{H}), 2.46(\mathrm{~d}, J=12.8 \mathrm{~Hz}), 2.33(\mathrm{bd}, J=13.3 \mathrm{~Hz}$, $1 \mathrm{H}), 2.26(\mathrm{dd}, J=11.1$ and $13.3 \mathrm{~Hz}, 1 \mathrm{H}), 2.21(\mathrm{~m}, 1 \mathrm{H}), 2.21(\mathrm{ddq}, 2.3,2.3$, and $7.2 \mathrm{~Hz}, 1 \mathrm{H})$, $1.80(\mathrm{~m}, 1 \mathrm{H}), 1.74(\mathrm{~s}, 3 \mathrm{H}), 1.50(\mathrm{~s}, 3 \mathrm{H}), 1.50(\mathrm{ddq}, J=3.9,10.3$, and $6.6 \mathrm{~Hz}, 1 \mathrm{H}), 1.39(\mathrm{dd}, \mathrm{J}$ $=11.1$ and $12.2 \mathrm{~Hz}, 1 \mathrm{H}), 1.27(\mathrm{~m}, 2 \mathrm{H}), 1.08(\mathrm{~d}, J=6.5 \mathrm{~Hz}, 3 \mathrm{H}), 0.99(\mathrm{~d}, J=6.5 \mathrm{~Hz}, 3 \mathrm{H})$, and $0.95(\mathrm{~d}, J=7.0 \mathrm{~Hz}, 3 \mathrm{H}) .{ }^{13} \mathrm{C} \mathrm{NMR}^{*}\left(125 \mathrm{MHz}, \mathrm{CD}_{3} \mathrm{OD}\right) \delta 172.9,141.6,134.4,134.3$, 132.0, 128.4, 113.5, 103.6, 96.6, 92.8, 83.9, 83.0, 81.4, 78.7, 78.4, 76.4, 72.7, 65.3, 62.7, $62.0,55.4,47.8,46.1,44.5,36.8,39.9,38.2,35.9,35.1,30.8,23.0,19.8,16.3,15.9,12.7$, and 6.8. *The weak carbamate carbonyl resonance at $\delta 161.1 \mathrm{ppm}$ reported for the natural sample of callipeltoside A was not observed. IR (neat) 3452, 3266, 2921, 2852, 1750, 1699, 1464,1358 , and $988 \mathrm{~cm}^{-1}$. HRMS (FAB) Calcd for $\left(\mathrm{C}_{35} \mathrm{H}_{48} \mathrm{ClNO}_{10}+\mathrm{Na}\right)^{+}:$700.2864, Found: 700.2883. TLC $\mathrm{R}_{\mathrm{f}}=0.5$, hexanes:EtOAc $=1: 1 .[\alpha]^{\mathrm{RT}}-53.8^{\circ}(\mathrm{c}=0.5, \mathrm{CHCl} 3)$.

\section{Supplementary Material}

Refer to Web version on PubMed Central for supplementary material.

\section{Acknowledgments}

These studies were supported by the National Cancer Institute (CA-76497) of the United States National Institutes of Health. We thank Mr. Aaron M. Johnson for his preparation of intermediates enroute to fragments 3 and 4 . 


\section{References}

(1). a) Zampella A, D'Auria MV, Minale L, Debitus C, Roussakis C. J. Am. Chem. Soc 1996;118:11085-11088. b) Zampella A, D'Auria V, Minale L. Tetrahedron 1997;53:3243-3248.

(2). Paterson I, Davies RDM, Marquez R. Angew. Chem. Int. Ed 2001;40:603-607.

(3). Total syntheses: a) Trost BM, Dirat O, Gunzner JL. Angew. Chem. Int. Ed 2002;41:841-843. b) Evans DA, Hu J, Burch JD, Jaeschke G. J. Am. Chem. Soc 2002;124:5654-5655. [PubMed: 12010035] c) Trost BM, Gunzner JL, Dirat O, Rhee YH. J. Am. Chem. Soc 2002;124:1039610415. [PubMed: 12197742] d) Paterson I, Davies RDM, Heimann AC, Marquez R, Meyer A. Org. Lett 2003;5:4477-4480. [PubMed: 14602029] e) Huang H, Panek JS. Org. Lett 2004;6:4383-4385. [PubMed: 15524489] f) Evans DA, Burch JD, Hu E, Jaeschke G. Tetrahedron 2008;64:4671-4699. g) Carpenter J, Northrup AB, Chung D, Wiener JJM, Kim S-G, MacMillan DWC. Angew. Chem. Int. Ed 2008;47:3568-3572.

(4). For a recent compilation of approaches to the synthesis of the macrolactone fragment, see ref $3 \mathrm{f}$.

(5). (a) Hoye TR, Zhao H. Org. Lett 1999;1:169-171. [PubMed: 10822555] (b) Zhao, H. PhD Thesis. University of Minnesota; 2000.

(6). Schwab P, France MB, Ziller JW, Grubbs RH. Angew. Chem. Int. Ed 1995;34:2039-2041.

(7). Danielson, ME. PhD Thesis. University of Minnesota; 2003.

(8). Scholl M, Ding S, Lee CW, Grubbs RH. Org. Lett 1999;1:953-956. [PubMed: 10823227]

(9). (a) Hoye TR, Jeffrey CS, Tennakoon MA, Wang J, Zhao H. J. Am. Chem. Soc 2004;126:1021010211. [PubMed: 15315410] (b) Wang X, Bowman EJ, Bowman BJ, Porco JA. Angew. Chem. Int. Ed 2004;43:3601-3605.(c) Hoye, TR.; Jeon, J. Methathesis Involving a Relay and Applications in Natural Product Synthesis. In: Cossy, J.; Arseniyadis, S.; Meyer, C., editors. Metathesis in Natural Product Synthesis. Wiley-VCH; Weinheim: 2010. p. 261-285.

(10). Schoening KU, Wittenberg R, Kirschning A. Synlett 1999;10:1624-1626.

(11). Sato M, Sugita Y, Abiko Y, Kaneko C. Tetrahedron: Asymmetry 1992;3:1157-1160.

(12). Guo J, Duffy KJ, Stevens KL, Dalko PI, Roth RM, Hayward MM, Kishi Y. Angew. Chem. Int. Ed 1998;37:187-192.

(13). Corey EJ, Bock MG, Kozikowski AP, Rama Rao AV, Floyd D, Lipshutz B. Tetrahedron Lett 1978;19:1051-1054.

(14). Schaus SE, Brandes BD, Larrow JF, Tokunaga M, Hansen KB, Gould AE, Furrow ME, Jacobsen EN. J. Am. Chem. Soc 2002;124:1307-1315. [PubMed: 11841300]

(15). Boeckman RK Jr. Pruitt JR. J. Am. Chem. Soc 1989;111:8286-8288.

(16). Marshall JA, Eidam PM. Org. Lett 2008;10:93-96. [PubMed: 18052287]

(17). Smith AB III, Verhoest PR, Minbiole KP, Schelhaas M. J. Am. Chem. Soc 2001;123:4834-4836. [PubMed: 11457294]

(18). Jones ERH, Stephenson JS, Turner WB, Whiting MC. J. Chem. Soc 1963:2048-2055.

(19). Rand CL, Van Horn DE, Moore MW, Negishi E. J. Org. Chem 1981;46:4096-4097.

(20). Smith AB, Barbosa J, Wong W, Wood JL. J. Am. Chem. Soc 1996;118:8316-8328.

(21). Boeckman RK Jr. Shao P, Wrobleski ST, Boehmler DJ, Heintzelman GR, Barbosa AJ. J. Am. Chem. Soc 2006;128:10572-10588. [PubMed: 16895426]

(22). Hoye TR, Danielson ME, May AE, Zhao H. Angew. Chem. Int. Ed 2008;47:9743-9746.

(23). a) Freiermuth B, Wentrup C. J. Org. Chem 1991;56:2286-2289. b) Birney DM, Wagenseller PE. J. Am. Chem. Soc 1994;116:6262-6270.

(24). Blanksby SJ, Ellison GB. Acc. Chem. Res 2003;36:255-263. [PubMed: 12693923]

(25). By this juncture, the configuration at C(13) in $\mathbf{1}$ had been established, ${ }^{2}$ so our subsequent studies described here all involved intermediates directed toward the $13 R$ configuration.

(26). Chlorocyclopropane sidechain syntheses: a) Olivo HF, Velazquez F, Trevisan HC. Org. Lett 2000;2:4055-4058. [PubMed: 11112642] b) Evans DA, Burch JD. Org. Lett 2001;3:503-505. [PubMed: 11178810]

(27). Evans DA, Hu E, Tedrow JS. Org. Lett 2001;3:3133-3136. [PubMed: 11574013] 
(28). a) Heathcock CH, McLaughlin M, Medina J, Hubbs JL, Wallace GA, Scott R, Claffey MM, Hayes CJ, Ott GR. J. Am. Chem. Soc 2003;125:12844-12849. [PubMed: 14558833] b) Tang B, Bray CD, Pattenden G. Tetrahedron Lett 2006;47:6401-6404. c) Ding F, Jennings MP. J. Org. Chem 2008;73:5965-5976. [PubMed: 18588348]

(29). Hoffman RW, Stuermer R. Chem. Ber 1994;127:2511-2518. 

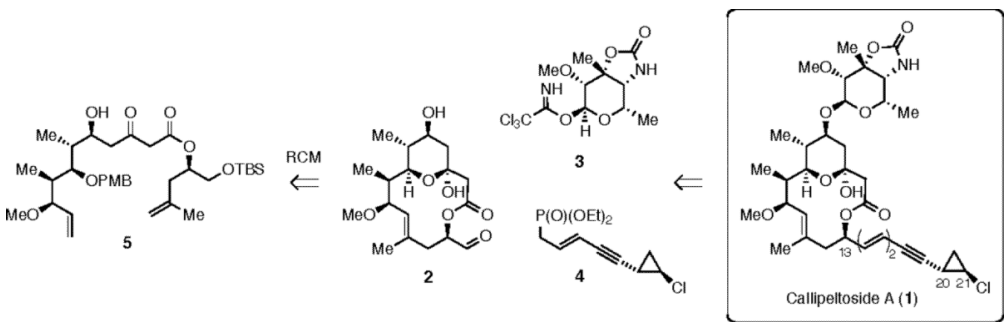

Scheme 1.

Callipeltoside Retrosynthesis. 


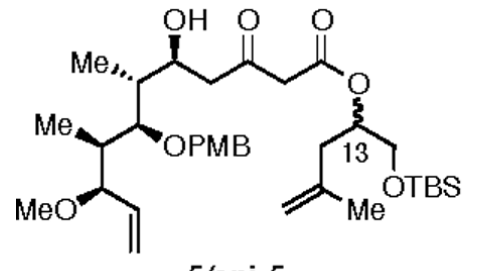

5/epi-5

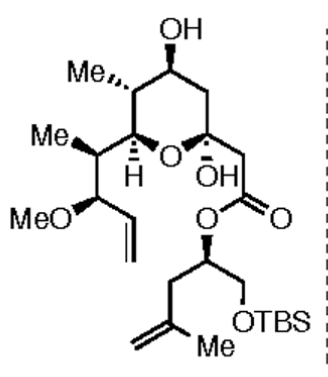

6

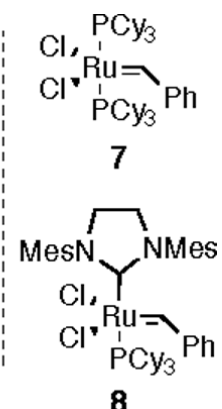

Scheme 2.

RCM Substrates. 

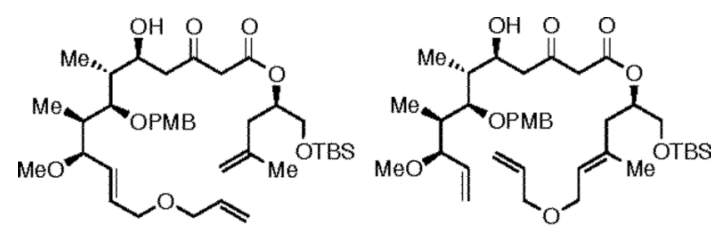

9

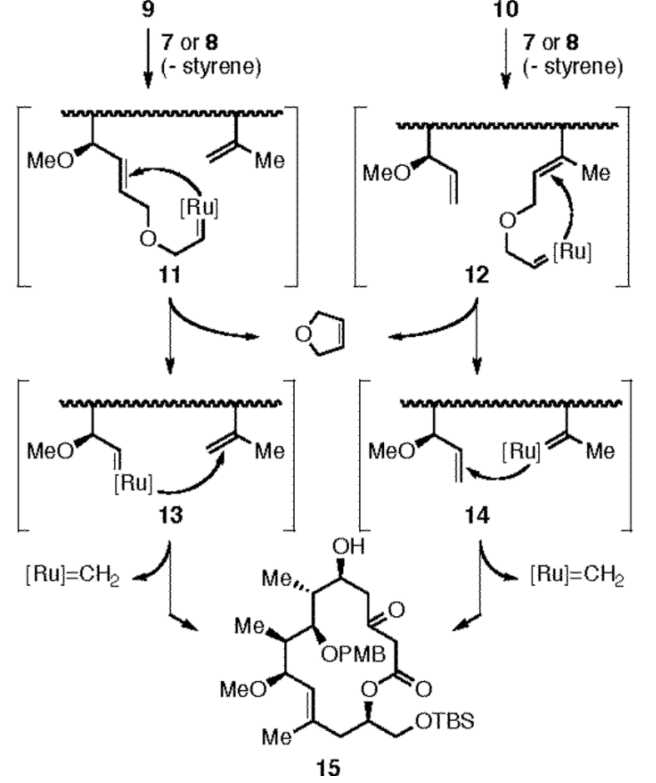

Scheme 3.

Relay Ring-Closing Metathesis. 


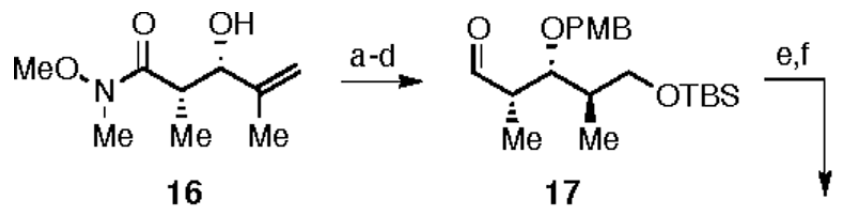

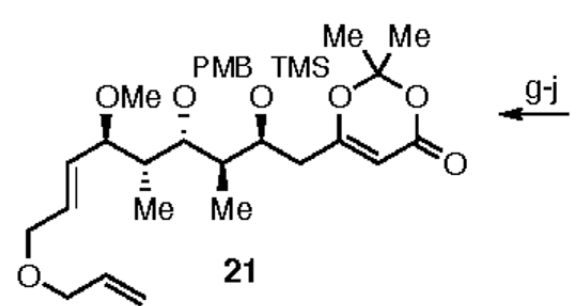

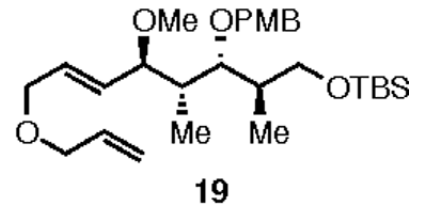

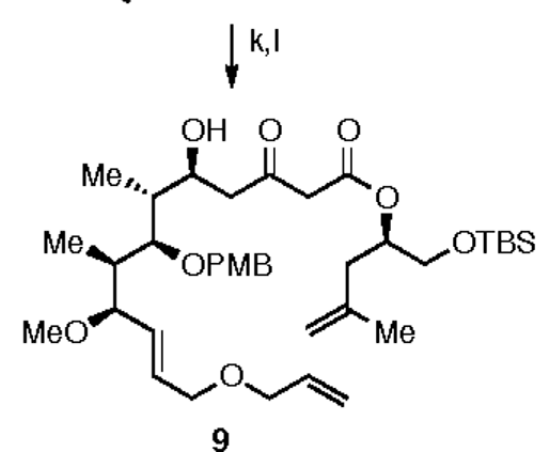

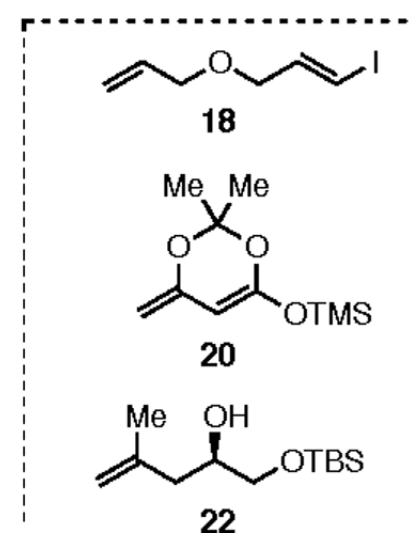

Scheme 4. Synthesis of Relay Substrate 9

a) PMB acetimidate, $\mathrm{CSA}, 92 \%$; b) 9-BBN, then $\mathrm{NaOH} / \mathrm{H}_{2} \mathrm{O}_{2}, 56 \%$; c) TBSCl, TEA, $60 \%$; d) DIBAL-H, 95\%; e) ${ }^{t} \mathrm{BuLi}$, then $\mathbf{1 8}, \mathrm{dr}=1: 1.5 ; \mathrm{SiO}_{2}$ separation; $22 \% / 33 \%$; f) MeI, $\mathrm{NaH}$, DMF, 64\%; g) TBAF, THF; h) DMP; i) 20, $\mathrm{BF}_{3} \bullet \mathrm{OEt}_{2}$; j) TMSCl, $65 \%$ over 4 steps; k) 22, benzene reflux; 1) PPTS, ACN 69\% over 2 steps. 

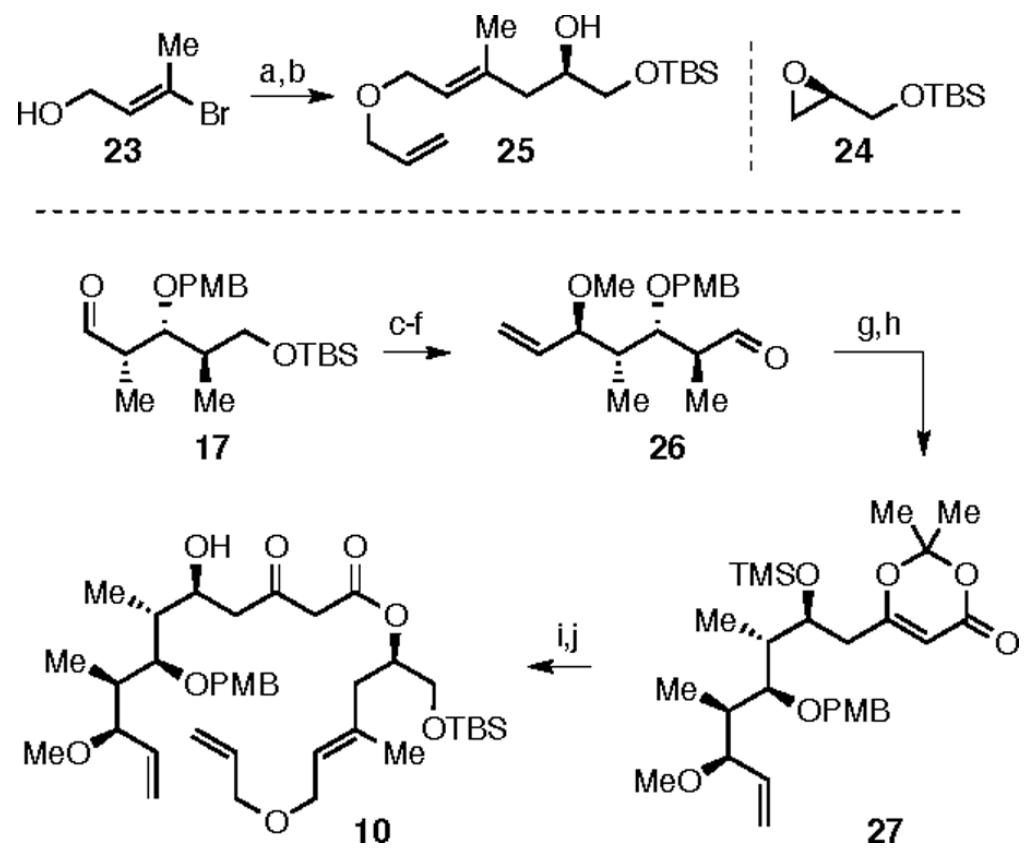

Scheme 5. Synthesis of Relay Substrate 10

a) allyl bromide, $\mathrm{NaH}, 81 \%$; b) ${ }^{\mathrm{t}} \mathrm{BuLi}, \mathrm{CuI}, \mathbf{2 4}$; c) vinylMgBr, $\mathrm{dr}=1: 1 ; \mathrm{SiO}_{2}$ separation; 34\%/34\%; d) MeI, NaH, DMF, 89\%; e) TBAF, THF, 87\%; f) DMP, 92\%; g) 20, $\mathrm{BF}_{3} \cdot \mathrm{OEt}_{2}$, 90\%; h) TMSCl, TEA, $65 \%$; i) 25, benzene reflux; j) PPTS, ACN, $77 \%$ over two steps. 

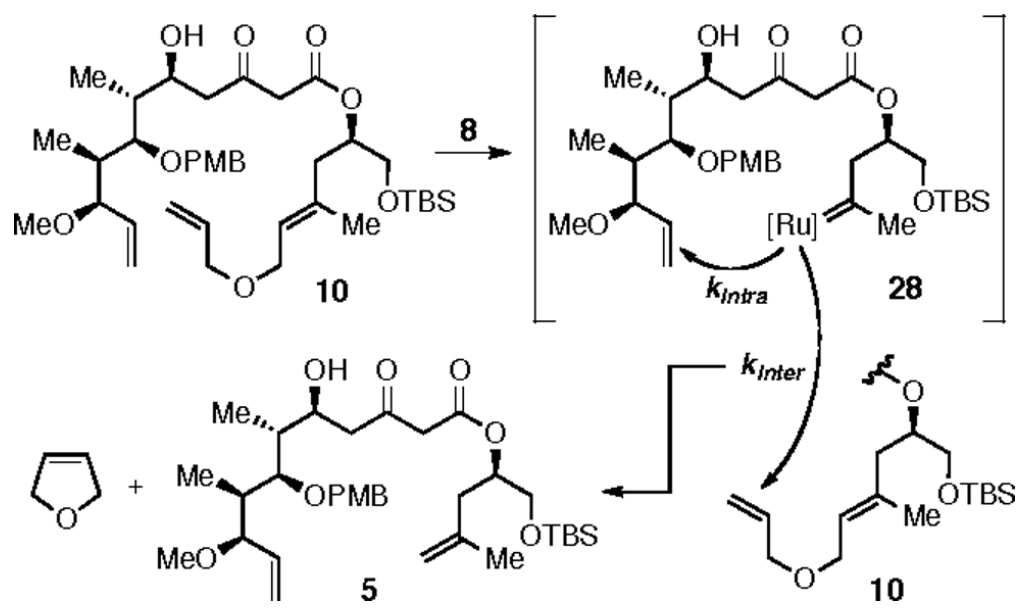

Scheme 6.

Truncation of Relay Substrate 10. 

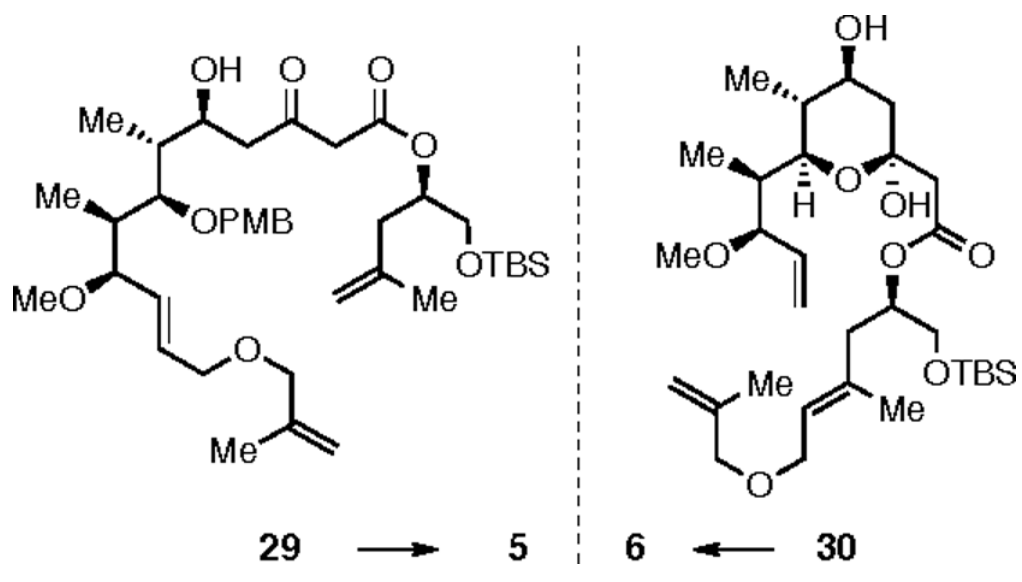

Scheme 7.

Additional RRCM Substrates. 


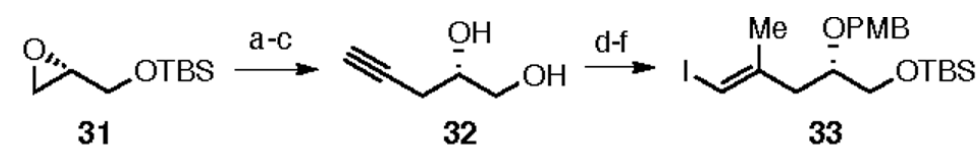

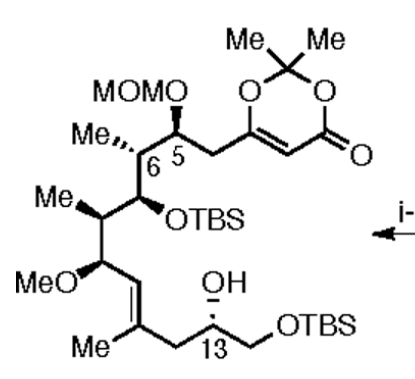

36
32

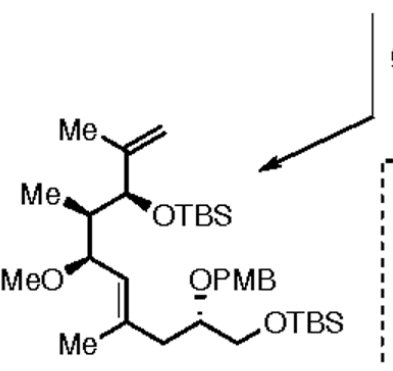

35 g,h

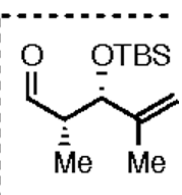

34

Scheme 8. Synthesis of Lactonization Precursor 38

a) Li-TMS-acetylide, $\mathrm{BF}_{3} \bullet \mathrm{OEt}_{2}$; b) $\mathrm{K}_{2} \mathrm{CO}_{3}, \mathrm{MeOH}$; c) $\mathrm{KF}, \mathrm{MeOH}$ reflux, $77 \%$ over 3 steps;

d) $\mathrm{AlMe}_{3}, \mathrm{ZrCp}_{2} \mathrm{Cl}_{2} ; \mathrm{I}_{2}, 65 \%$; e) $\mathrm{TBSCl}$, imidazole, $72 \%$; f) PMB acetimidate, CSA, 82\%;

g) n-BuLi, 34, dr = 1:1; h) MeI, $\mathrm{NaH}, 80 \%$; i) 9-BBN, then $\mathrm{NaOH} / \mathrm{H}_{2} \mathrm{O}_{2}, 99 \%$; j) DMP, $79 \%$; k) 20, $\mathrm{BF}_{3} \cdot \mathrm{OEt}_{2}, 70 \%$; 1) $\mathrm{MOMCl}, 95 \%$; m) DDQ, $100 \%$. 


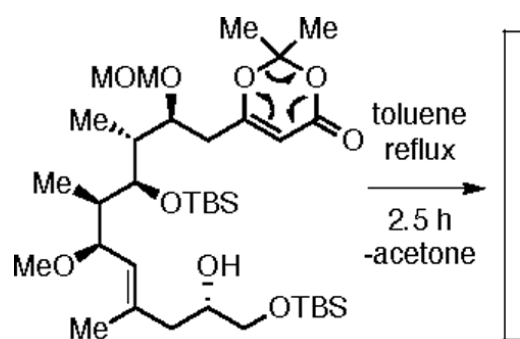

36
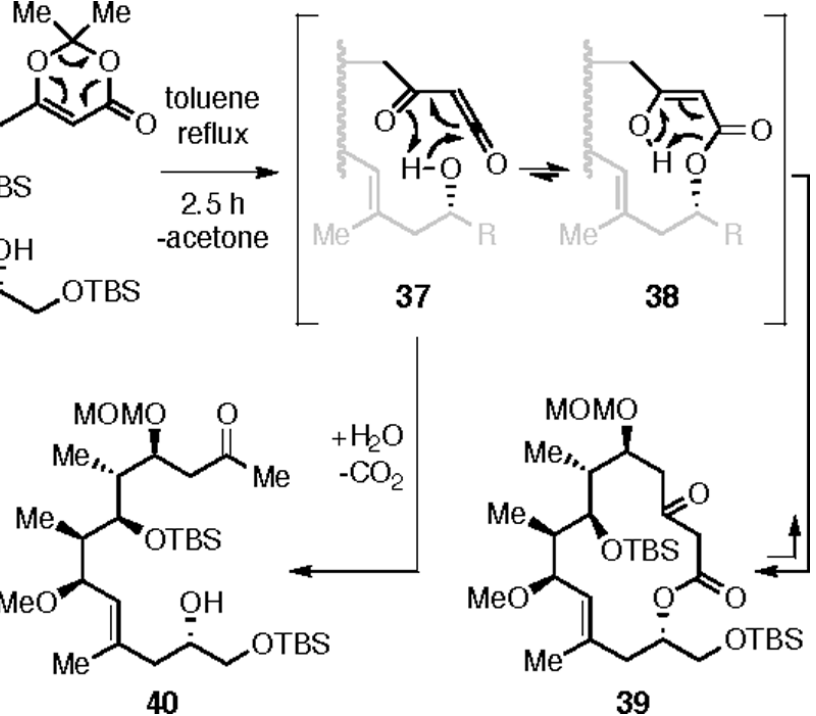

Scheme 9.

Boeckman Cyclization and Competitive Hydrolysis of 36. 


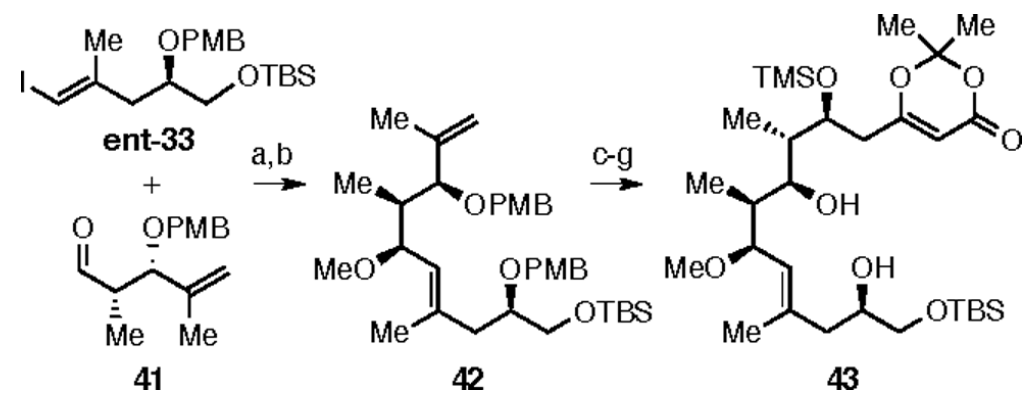

Scheme 10. Synthesis of Dual Macrolactonizion/Pyran-Hemiketal Precursor 43

Reagents and conditions: (a) ${ }^{n} \mathrm{BuLi}$, ent-33; then 41, $\mathrm{dr}=1: 1, \mathrm{SiO}_{2}$ separation, 34\%/34\%;

(b) $\mathrm{MeI}, \mathrm{NaH}, 80 \%$; (c) 9-BBN, then $\mathrm{NaOH} / \mathrm{H}_{2} \mathrm{O}_{2}, 70 \%$; (d) DMP, 76\%; (e) $20, \mathrm{BF}_{3} \cdot \mathrm{OEt}_{2}$, 90\%; (f) TMSCl; g) DDQ, 78\% over 2 steps. 


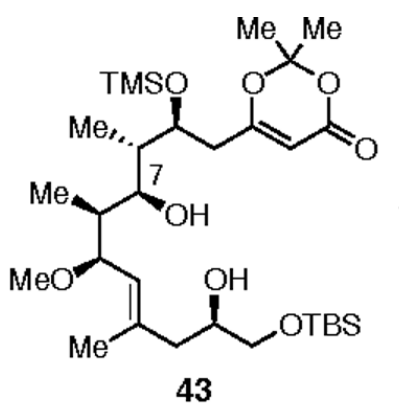

43

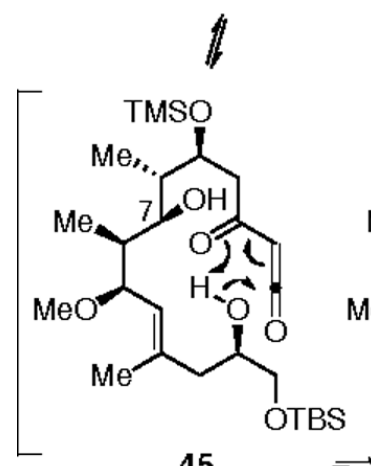

45

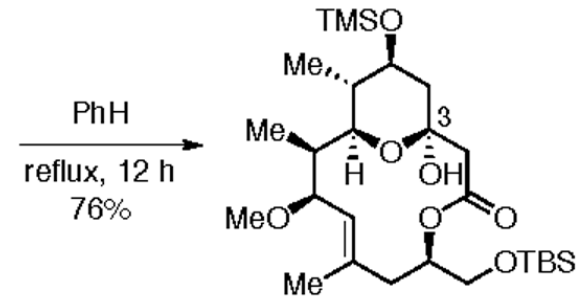

44

1

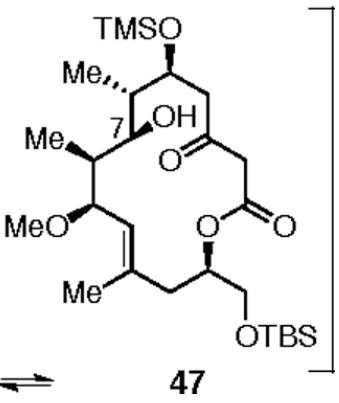

Scheme 11.

Formation of Pyran-Hemiketal 44. 


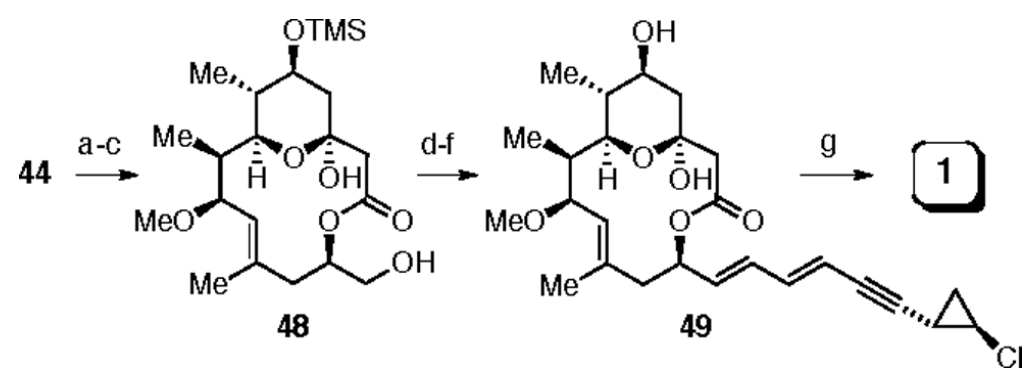

Scheme 12. Completion of the Synthesis

Reagents and conditions: (a) TBAF, THF; (b) TMSCl, TEA, DCM; (c) $\mathrm{NaHCO}_{3}, \mathrm{MeOH}$, $70 \%$ over 3 steps; (d) $\mathrm{SO}_{3} \bullet$ pyr, DMSO, TEA; (e) 4, LiHMDS, THF; (f) TBAF, THF, 54\% over 3 steps; (g) 5, TMSOTf, DCM, 70\%. 